\title{
Optimum tuning of passive Tuned Mass Dampers for the mitigation of pulse-like responses
}

\author{
Jonathan SALVI ${ }^{1}$, Egidio RIZZI ${ }^{1, *}$, Emiliano RUSTIGHI ${ }^{2}$, Neil S. FERGUSON ${ }^{2}$ \\ ${ }^{1}$ Department of Engineering and Applied Sciences, University of Bergamo \\ viale G. Marconi 5, I-24044 Dalmine (BG), Italy \\ E-mail: jonathan.salvi@unibg.it; egidio.rizzi@unibg.it \\ ${ }^{*}$ Corresponding Author, Professor
${ }^{2}$ Institute of Sound and Vibration Research, University of Southampton
Highfield, Southampton SO17 1BJ, UK
E-mail: er@isvr.soton.ac.uk; nsf@isvr.soton.ac.uk

\begin{abstract}
Tuned Mass Dampers (TMDs) are typically introduced and calibrated as natural passive control devices for the vibration mitigation of the steady-state response of primary structures subjected to persistent excitations. Otherwise, this work investigates the optimum tuning of TMDs towards minimising the transient structural response. Specifically, a single-degreeof-freedom system is considered as a primary structure, with added TMD, subjected to pulse-like excitations. First, the system is analytically analysed, within the time domain, for unit impulse base displacement, through Laplace transform. Then, the tuning process is numerically explored by an optimisation procedure focused on an average response index, to extract the optimum condition towards best TMD calibration. The efficiency of the proposed control device is then assessed and demonstrated through further post-tuning numerical tests, by considering as dynamic loadings: first, a time unit impulse base displacement, coherent with the source description above; second, different pulse-like excitations, to detect the effectiveness of the so-conceived TMD for generic ideal shock actions; third, a set of nonstationary earthquake excitations, to enquire the achievable level of seismic isolation. It is shown that this leads to a consistent passive TMD in such a transient excitation context, apt to mitigate the average response. Additionally, the present tuning forms a necessary optimum background for a possible upgrade to a hybrid TMD, with the potential addition of an active controller to the so-optimised TMD, to achieve even further control performance, once turned on, specifically for abating the peak response, too.
\end{abstract}

Keywords: Tuned Mass Damper (TMD); Optimum TMD Tuning; Unit Impulse Excitation; Pulse-like shock actions; Seismic response reduction. 


\section{Introduction}

In this study, the optimisation of a Tuned Mass Damper control device under transient impulse excitation is developed, as an innovative part of a wider research project on the general dynamic vibration absorber tuning concept [18,51] and on the specific TMD optimisation process [37-39]. Particularly on TMD calibration, while TMDs have been optimised for reference loadings in [38] and for earthquake excitation in [37,39], in this paper, and in companion work [36], the innovative scenario of TMD tuning under impulse excitation is considered, here by proposing a purely passive TMD, apt to control the average structural response along the whole time window of analysis, there by outlining a hybrid TMD, relying on the present background tuning, apt to further control also the peak structural response, if made available and once turned on. That may consider the further upgrade of the present passive control device to a hybrid version, whereby an active controller may be added to the optimised passive TMD as here derived. Thus, the present work forms a necessary background reference for such a possible active update of the passive vibration control device here under investigation. A compact review on the use of different types of passive, active and semiactive control systems, with focus on passive devices for seismic response reduction, a main application perspective of the whole present research investigation, may be found in [28]. Recent studies on advanced TMD technologies with energy harvesting/dissipation have lately appeared on this journal in [21,22].

The first documented proposal of a TMD is likely represented by the patent of Frahm (1911), and has been followed by different initial fundamental studies (Ormondroyd and Den Hartog, 1928; Brock, 1946), as finally codified in Den Hartog book [10], which outlined the theoretical bases and formulae for the optimum design of the TMD parameters, assuming external loading as a harmonic excitation, acting as a force on an undamped primary structure. Afterwards, many works have focused on the optimum tuning of TMDs, in order to deepen the tuning knowledge on different response indices and dynamic excitations. In particular, the usual framework of such studies has considered a Single-Degree-Of-Freedom (SDOF) damped primary structure, with added TMD, subjected to either harmonic $[14,34,35]$ or white noise $[6,19]$ excitations, acting as a force on the primary structure or as a base motion [4,20,48]. In general, passive TMDs appear to be usually characterised by a narrow operating frequency range and a high sensitivity to possible harmful effects of the so-called de-tuning, i.e. the loss of tuning due to changes in the structural features, which may compromise the overall performance of the control device. Also, the TMD parameters appear to be at least slightly dependent on the characteristics of the applied dynamic loading [7,23,41,42]. This issue motivated different investigations based, inter alia, on the modelling of real excitation cases, such as for random [43], wind [3,27] or seismic $[2,8,11,12,24-26,44]$ actions.

In the case of impulse excitation, as here addressed, a passive TMD may generally be considered as being not significantly effective in reducing the structural response [1], even if several important issues must still be deepened in this sense, as outlined in recent contributions within this specific later TMD field [25,33], which seems to have received less attention in the dedicated literature, than for the more consolidated contexts above. For instance: expected effectiveness of TMD as the pulse action duration shortens; use of $\mathrm{H}_{\infty}$ (peak) or other, e.g. $\mathrm{H}_{2}$ (average), norms, looking at peak or average response index mitigation, respectively; effectiveness of TMD at increasing mass ratio, which may be pushed to high values; difference between the use of analytical or codified pulse-like ground motions vs. real earthquake pulse ground motions; overall powerfulness of TMD insertion for mitigating pulse-like action responses and importance of the specific tuning concerning that. These open issues indeed motivate for further investigations on this peculiar subject of TMD calibration, as handled within the paper.

The present fundamental analytical/numerical study, prior to possible subsequent foreseen experimental validation and application scenarios, is placed within such a specific TMD tun- 
ing context, by concerning the underlying theoretical optimum tuning of an added TMD when the primary structure is subjected to shock loading. While here experimental aspects are not directly considered, perspectives in that sense may be addressed by following literature contributions on this specific TMD tuning and related subjects. For instance, recent experimental studies have inspected the response of SDOF or Multi-Degree-Of-Freedom (MDOF) primary structures equipped with TMDs. In [9], multiple mass dampers were earlier tested to suppress the seismic responses of a scaled three-storey building by vibration tests on a shaking table, under white noise excitation and two different scaled earthquakes, showing the multiple damper system to being rather effective, and likely superior to a single TMD, in mitigating the floor accelerations. In [50], a proposal of TMD tuning with a small required stroke length for baseisolated or high-rise structures was experimentally assessed by small-scale model experimental setups, as subjected to a time scaled long period impulsive excitation and a shaking table earthquake excitation experiment, indicating the TMD control system to displaying a high potential for seismic response control or retrofit of structures, even when a limited space is made available to accommodating TMDs. In [49], the seismic response reduction of an offshore platform by the application of a specific TMD system was explored on a scaled prototype (SDOF+TMD) model, by an experimental study under different earthquakes, demonstrating that the proposed TMD system can effectively suppress the earthquake stimulus on the response of the offshore platform. Further, within the specific presence of dynamic Soil-Structure-Interaction (SSI) effects, recent experimental investigations were made by [15-17], where interesting centrifuge tests were conducted on MDOF multi-storey structural models. In [15], it was shown that the TMD tuning based on the properties of the soil-structure system may lead to doubling the improvement gain in reducing the original peak response, while a great response magnification may be recorded for a de-tuned TMD neglecting SSI effects, thus pointing out to the importance of making a TMD tuning on the soil-structure system, and with an appropriate description of SSI effects, as theoretically investigated in [40]. In [16], the specific issue of storey TMD positioning was enquired, showing this to possibly playing a role on the overall TMD effectiveness, within SSI, and potentially lead, if non-optimal, to detuning-similar effects. In [17], Structure-Soil-Structure Interaction (SSSI) of adjacent structures retrofitted by TMDs was additionally investigated, showing that harmful effects linked to detuning may become even higher.

In the present paper, an ideal structural system composed of a linear damped SDOF primary structure and a linear TMD added on it, and subjected to a true unit impulse excitation acting as base displacement on the supported structure, has been considered first and assumed as a main reference case for the TMD methodological tuning purposes. Several features presented and discussed within this theoretical study hold true also when the unit impulse excitation may act as a force on the primary structure, which represents a complementary interesting scenario, for instance in terms of recorded transient response, vibration control and potential of reducing the structural response, specifically in terms of average response indices, and effective adoption of a TMD in this context. Subsequently, several post-tuning pulse-like excitations are numerically analysed, and the TMD efficiency is fully assessed and confirmed in that context, nearing real applications. Finally, implications in the seismic engineering range are also successfully explored, by considering the post-tuning system response to instances of a set of earthquake excitations and by observing the achieved seismic response reduction, in terms of both peak and average response indices all along the transient time window of analysis, the latter being best and well controlled by the conceived TMD device.

This study is organised as follows. First, the structural context is explained in detail, and the related dynamic response is analytically obtained in closed form (Section 2). In Section 3, the numerical optimisation of a passive TMD is developed, showing all the possible application benefits. In particular, the tuning methodology is presented, with a specific consideration of the adopted objective function and of the implemented numerical algorithm. A single set of 
mechanical parameters has been assumed for the primary structure so far, in order to first investigate the efficiency of the tuning procedure. However, besides slight differences in the numerical values, which may depend on the assumed structural parameters, the optimisation principles here derived display a rather general validity, and could therefore be applied to a wide range of engineering applications. The so-obtained results are mainly composed of the optimum TMD parameters under true unit impulse base excitation (Section 4) and of the achieved dynamic response reduction (Section 5), illustrated in the form of appropriate plots and tables. Besides for the unit impulse base displacement, which plays the role of the main dynamic excitation considered in this work, several real pulse-like excitations have been then considered in further post-tuning trials, together with different reference tuning methods (Appendix A), so that to assess the validity of the proposed impulse-tuned TMD also for generic ideal shock actions (Appendix B), and finally for real random earthquake excitations. A resuming discussion on the results obtained by the whole study, focusing on its various technical details, is then outlined in Section 6. Finally, Section 7 states the closing thesis of the present research investigation.

\section{Structural and dynamic context}

\subsection{SDOF primary structure}

A reference SDOF system is considered, initially at rest and subjected to a true unit impulse excitation at $t=0$, which may be ideally defined by a Dirac delta function $\delta(t)$. Particularly, such an impulse excitation has been considered to be acting as a base displacement $x_{g}(t)$ (Fig. 1$)$ :

$$
x_{g}(t)=\bar{X}_{g} \delta(t)
$$

where $\bar{X}_{g}$ denotes the constant (unit) displacement impulse of the base excitation, here nominally assumed as $\bar{X}_{g}=0.01 \mathrm{~ms}$.

\section{Figure 1}

The assumption of ground motion in terms of absolute base displacement shall provide two main considerable advantages: a) it allows for an easier formulation than that in the case of a relative ground acceleration; b) it is more suitable from the viewpoint of real dynamic response measuring, which turns out to be feasible when focused on the detection of absolute coordinates rather than on the relative motion between the masses of the system.

These reasons may even look more important in the case of a MDOF primary structure, which may reflect real engineering contexts and represent a generalisation scenario of the present SDOF analysis, maybe also involving the adoption of Multiple TMDs [5,7,9] or the issue of TMD positioning along the structure [16] and of structural system identification related contexts [13, 29-31], possibly in the contextual presence of vibration control devices [47], and/or of specific characteristic structural effects, like Soil-Structure Interaction [15-17,32, 40].

The mechanical parameters of such a SDOF linear system are: mass $m_{1}$, constant stiffness $k_{1}$ and viscous damping coefficient $c_{1}$. In this sense, for the present study, the following nominal values of the structural parameters have been fixed: $m_{1}=100 \mathrm{~kg}, k_{1}=10000 \mathrm{~N} / \mathrm{m}$. Notice however that, in case of different structural parameters, the methodology and related results here outlined are characterised by a general validity. Different amounts of structural damping have been considered, by focusing then on typical (subcritical) values for civil structural systems, apt to already challenge the further application of a TMD controller (higher challenge of TMD effectiveness being expected at increasing inherent structural damping). 
The associated natural angular frequency $\omega_{1}$ and damping ratio $\zeta_{1}$ of the primary structure are classically defined as follows:

$$
\omega_{1}=\sqrt{\frac{k_{1}}{m_{1}}}, \quad \zeta_{1}=\frac{c_{1}}{c_{1, c r}}=\frac{c_{1}}{2 \sqrt{k_{1} m_{1}}}=\frac{c_{1}}{2 \omega_{1} m_{1}}
$$

where $c_{1, c r}$ is the critical damping coefficient of the primary system. Then, inherent structural damping ratios in the order up to $\zeta_{1}=5 \%$ will be considered in the following investigation analysis, thus setting an increasing challenge for TMD effectiveness.

The dynamic behaviour of such a primary structure is ruled by the following equation of motion in terms of absolute displacement DOF $x_{1}(t)$ (Fig. 1):

$$
m_{1} \ddot{x}_{1}(t)+c_{1} \dot{x}_{1}(t)+k_{1} x_{1}(t)=c_{1} \bar{X}_{g} \dot{\delta}(t)+k_{1} \bar{X}_{g} \delta(t)
$$

or, in terms of the characteristic system parameters, i.e. by dividing all components by $m_{1}$ and by the definitions above:

$$
\ddot{x}_{1}(t)+2 \zeta_{1} \omega_{1} \dot{x}_{1}(t)+\omega_{1}^{2} x_{1}(t)=2 \zeta_{1} \omega_{1} \bar{X}_{g} \dot{\delta}(t)+\omega_{1}^{2} \bar{X}_{g} \delta(t) .
$$

The analytical expressions of the involved indices and response norms $\left(\mathrm{H}_{2}, \mathrm{H}_{\infty}\right)$ have been obtained in closed form, as below reported.

\subsection{SDOF primary structure response indices}

In this section, the explicit expressions of the $\mathrm{H}_{\infty}$ and $\mathrm{H}_{2}$ norms of SDOF primary structure displacement response $x_{1}(t)$ to unit impulse base displacement $x_{g}(t)=\bar{X}_{g} \delta(t)$ are presented.

The time response of the primary structure is obtained through a pair of Laplace Transforms. First, equation of motion (4) (Section 2.1) is transformed in frequency Laplace variable $s$, with homogeneous conditions, consistently with the hypothesis of system initially at rest:

$$
\left[s^{2}+s 2 \zeta_{1} \omega_{1}+\omega_{1}^{2}\right] X_{1}(s)=s 2 \zeta_{1} \omega_{1} \bar{X}_{g}+\omega_{1}^{2} \bar{X}_{g} \quad \Rightarrow \quad Z_{1}(s) X_{1}(s)=F_{1}(s)
$$

where $Z_{1}(s)$ is the impedance of the system, $X_{1}(s)$ is the degree of freedom response and $F_{1}(s)$ is the Laplace transform of the dynamic excitation. Thus, the transform of the dynamic response of the primary structure, in terms of displacement, as a function of $s$, can be expressed as:

$$
X_{1}(s)=Z_{1}(s)^{-1} F_{1}(s)=H_{1}(s) F_{1}(s)
$$

where $H_{1}(s)=Z_{1}(s)^{-1}$ is the receptance (or transfer function) of the system. Hence, one obtains:

$$
X_{1}(s)=\bar{X}_{g} \frac{s 2 \zeta_{1} \omega_{1}+\omega_{1}^{2}}{s^{2}+s 2 \zeta_{1} \omega_{1}+\omega_{1}^{2}}=\bar{X}_{g} \frac{g 2 \zeta_{1}+1}{g^{2}+g 2 \zeta_{1}+1} .
$$

where $g=s / \omega_{1}$.

The inverse Laplace transform of this relation provides the following analytical expression of the time displacement of the SDOF primary structure:

$$
x_{1}(t)=\bar{X}_{g} \omega_{1} \frac{\mathrm{e}^{-\zeta_{1} \omega_{1} t}}{\sqrt{1-\zeta_{1}^{2}}} \cdot\left[\left(1-2 \zeta_{1}^{2}\right) \sin \left(\omega_{1, d} t\right)+2 \zeta_{1} \sqrt{1-\zeta_{1}^{2}} \cos \left(\omega_{1, d} t\right)\right]
$$

where $\omega_{1, d}=\omega_{1} \sqrt{1-\zeta_{1}^{2}}$, with the following velocity derivative:

$$
\dot{x}_{1}(t)=\bar{X}_{g} \omega_{1}^{2} \frac{\mathrm{e}^{-\zeta_{1} \omega_{1} t}}{\sqrt{1-\zeta_{1}^{2}}} \cdot\left[-\zeta_{1}\left(3-4 \zeta_{1}^{2}\right) \sin \left(\omega_{1, d} t\right)+\sqrt{1-\zeta_{1}^{2}}\left(1-4 \zeta_{1}^{2}\right) \cos \left(\omega_{1, d} t\right)\right] .
$$


Thus, response $x_{1}(t)$ to $x_{g}(t)=\bar{X}_{g} \delta(t)$ may be interpreted as a damped free vibration to nonhomogeneous initial conditions at time $0^{+}$as:

$$
x_{1,0^{+}}=\bar{X}_{g} \frac{c_{1}}{m_{1}}=\bar{X}_{g} \omega_{1} 2 \zeta_{1} ; \quad \dot{x}_{1,0^{+}}=\bar{X}_{g}\left(\frac{k_{1}}{m_{1}}-\left(\frac{c_{1}}{m_{1}}\right)^{2}\right)=\bar{X}_{g} \omega_{1}^{2}\left(1-4 \zeta_{1}^{2}\right) .
$$

Particular interest regards two response indices obtainable from Eq. (8), namely the $\mathrm{H}_{2}$ and $\mathrm{H}_{\infty}$ norms of the primary structure displacement, which refer to the overall and the peak response, respectively. General $\mathrm{H}_{i}(1<i<\infty)$ and $\mathrm{H}_{2}, \mathrm{H}_{\infty}$ norms over time window of analysis $T_{a}$ can be classically defined as:

$$
\left\|x_{1}(t)\right\|_{i}:=\left(\int_{0}^{T_{a}}|x(t)|^{i} \mathrm{~d} t\right)^{\frac{1}{i}} ; \quad\left\|x_{1}(t)\right\|_{2}:=\sqrt{\int_{0}^{T_{a}}\left|x_{1}(t)\right|^{2} \mathrm{~d} t}, \quad\left\|x_{1}(t)\right\|_{\infty}:=\max _{t}\left|x_{1}(t)\right| .
$$

The $\mathrm{H}_{\infty}$ norm is evaluated first. By setting the first derivative in Eq. (9) equal to zero, such a stationary condition marks peak time $\bar{t}$ where the peak displacement is obtained:

$$
\bar{t}=\frac{1}{\omega_{1, d}} \arctan \left(\frac{\sqrt{1-\zeta_{1}^{2}}\left(1-4 \zeta_{1}^{2}\right)}{\zeta_{1}\left(3-4 \zeta_{1}^{2}\right)}\right)
$$

The substitution of Eq. (12) into Eq. (8) provides the expression of the peak displacement, or $\mathrm{H}_{\infty}$ norm displacement:

$$
\left\|x_{1}(t)\right\|_{\infty}=\bar{X}_{g} \omega_{1} \mathrm{e}^{-\frac{\zeta_{1}}{\sqrt{1-\zeta_{1}^{2}}} \arctan \left(\frac{\sqrt{1-\zeta_{1}^{2}}\left(1-4 \zeta_{1}^{2}\right)}{\zeta_{1}\left(3-4 \zeta_{1}^{2}\right)}\right)}
$$

which plays an important role in the tuning process, since the magnitude of the peak displacement is one of the main objectives within the control of the system transient response.

Then, the $\mathrm{H}_{2}$ norm of $x_{1}(t)$ is evaluated as well, by substituting Eq. (8) into Eq. (11):

$$
\begin{aligned}
\left\|x_{1}(t)\right\|_{2}=\frac{\bar{X}_{g} \sqrt{\omega_{1}}}{\sqrt{4 \zeta_{1}\left(1-\zeta_{1}^{2}\right)} \cdot(}\left(1+4 \zeta_{1}^{2}\right)\left(1-\zeta_{1}^{2}\right)+ & \\
-\mathrm{e}^{-2 \zeta_{1} \omega_{1} T_{a}}[1 & +\zeta_{1}\left(1-4 \zeta_{1}^{2}\right) \sqrt{1-\zeta_{1}^{2}} \sin \left(2 \omega_{1, d} T_{a}\right)+ \\
& \left.\left.+\zeta_{1}^{2}\left(3-4 \zeta_{1}^{2}\right) \cos \left(2 \omega_{1, d} T_{a}\right)\right]\right)^{1 / 2}
\end{aligned}
$$

\subsection{Structural system with TMD}

The structural system composed of a SDOF primary structure and a TMD added on top of it, assumed as a benchmark model in the present study, is further represented as a 2DOF linear system in Fig. 2. Similarly as for the primary structure alone, the TMD parameters are mass $m_{2}$, constant stiffness $k_{2}$ and viscous damping coefficient $c_{2}$, while relevant natural angular frequency $\omega_{2}$ and damping ratio $\zeta_{2}$ are consistently defined as in Eq. (2):

$$
\omega_{2}=\sqrt{\frac{k_{2}}{m_{2}}}, \quad \zeta_{2}=\frac{c_{2}}{c_{2, c r}}=\frac{c_{2}}{2 \sqrt{k_{2} m_{2}}}=\frac{c_{2}}{2 \omega_{2} m_{2}}
$$


where $c_{2, c r}$ is the critical damping coefficient of the added TMD. Further two parameters are then classically introduced for the purposes of the TMD tuning process, i.e. TMD mass ratio $\mu$ and frequency ratio $f$ (while TMD damping ratio $\zeta_{2}$ keeps defined as in Eq. (15)):

$$
\mu=\frac{m_{2}}{m_{1}}, \quad f=\frac{\omega_{2}}{\omega_{1}}=\sqrt{\frac{1}{\mu} \frac{k_{2}}{k_{1}}} .
$$

Figure 2

The equations of motion of the considered 2DOF linear structural system can be stated in classical matrix form as follows:

$$
\mathbf{M} \ddot{\mathbf{x}}(t)+\mathbf{C} \dot{\mathbf{x}}(t)+\mathbf{K x}(t)=\mathbf{F}(t)
$$

where

$$
\mathbf{M}=\left[\begin{array}{cc}
m_{1} & 0 \\
0 & m_{2}
\end{array}\right], \mathbf{C}=\left[\begin{array}{cc}
c_{1}+c_{2} & -c_{2} \\
-c_{2} & c_{2}
\end{array}\right], \mathbf{K}=\left[\begin{array}{cc}
k_{1}+k_{2} & -k_{2} \\
-k_{2} & k_{2}
\end{array}\right] ; \quad \mathbf{x}(t)=\left[\begin{array}{l}
x_{1}(t) \\
x_{2}(t)
\end{array}\right]
$$

denote the structural matrices of mass, viscous damping and elastic stiffness, respectively, and vector of absolute displacements $\mathbf{x}(t)$, with associated velocities $\dot{\mathbf{x}}(t)$ and accelerations $\ddot{\mathbf{x}}(t)$.

Vector $\mathbf{F}(t)$, which represents the external dynamic excitation, for the case of unit impulse base displacement takes the form:

$$
\mathbf{F}(t)=\left[\begin{array}{c}
c_{1} \dot{x}_{g}(t)+k_{1} x_{g}(t) \\
0
\end{array}\right]=\left[\begin{array}{c}
c_{1} \bar{X}_{g} \dot{\delta}(t)+k_{1} \bar{X}_{g} \delta(t) \\
0
\end{array}\right]
$$

For the purposes of the present study, vectors and matrices in Eqs. (18), (19) may be rewritten in terms of structural and TMD parameters, by dividing all terms by $m_{1}$, leading to the following array replacements:

$$
\begin{aligned}
& \mathbf{M} \rightarrow\left[\begin{array}{ll}
1 & 0 \\
0 & \mu
\end{array}\right], \quad \mathbf{C} \rightarrow\left[\begin{array}{cc}
2 \zeta_{1} \omega_{1}+2 \zeta_{2} \omega_{2} \mu & -2 \zeta_{2} \omega_{2} \mu \\
-2 \zeta_{2} \omega_{2} \mu & 2 \zeta_{2} \omega_{2} \mu
\end{array}\right], \quad \mathbf{K} \rightarrow\left[\begin{array}{cc}
\omega_{1}^{2}+\omega_{2}^{2} \mu & -\omega_{2}^{2} \mu \\
-\omega_{2}^{2} \mu & \omega_{2}^{2} \mu
\end{array}\right], \\
& \mathbf{F}(t) \rightarrow\left[\begin{array}{c}
2 \zeta_{1} \omega_{1} \bar{X}_{g} \dot{\delta}(t)+\omega_{1}^{2} \bar{X}_{g} \delta(t) \\
0
\end{array}\right] .
\end{aligned}
$$

The analytical expressions of the corresponding dynamic response indices are then derived as follows.

\subsection{Dynamic response of the structural system (SDOF primary structure + TMD)}

As for the SDOF system, the response of the considered structural system in the time domain is obtained by a pair of Laplace transforms. Firstly, the equations of motion above are Laplace transformed:

$$
\left[\begin{array}{ll}
Z_{11}(s) & Z_{12}(s) \\
Z_{21}(s) & Z_{22}(s)
\end{array}\right]\left[\begin{array}{l}
X_{1}(s) \\
X_{2}(s)
\end{array}\right]=\left[\begin{array}{l}
F_{1}(s) \\
F_{2}(s)
\end{array}\right] \Rightarrow \mathbf{Z}(s) \mathbf{X}(s)=\mathbf{F}(s)
$$


where $\mathbf{Z}(s)$ is the impedance matrix, $\mathbf{X}(s)$ is the vector of degrees of freedom and $\mathbf{F}(s)$ is the excitation vector. In particular, impedance matrix $\mathbf{Z}(s)$ takes the following form:

$$
\begin{aligned}
\mathbf{Z}(s) & =\left[\begin{array}{ll}
Z_{11}(s) & Z_{12}(s) \\
Z_{21}(s) & Z_{22}(s)
\end{array}\right] \\
& =\left[\begin{array}{cc}
s^{2}+s\left(2 \zeta_{1} \omega_{1}+2 \zeta_{2} \omega_{2} \mu\right)+\left(\omega_{1}^{2}+\omega_{2}^{2} \mu\right) & -s 2 \zeta_{2} \omega_{2} \mu-\omega_{2}^{2} \mu \\
-s 2 \zeta_{2} \omega_{2} \mu-\omega_{2}^{2} \mu & s^{2} \mu+s 2 \zeta_{2} \omega_{2} \mu+\omega_{2}^{2} \mu
\end{array}\right]= \\
& =\left[\begin{array}{cc}
g^{2}+g 2\left(\zeta_{1}+\zeta_{2} f \mu\right)+\left(1+f^{2} \mu\right) & -g 2 \zeta_{2} f \mu-f^{2} \mu \\
-g 2 \zeta_{2} f \mu-f^{2} \mu & g^{2} \mu+g 2 \zeta_{2} f \mu+f^{2} \mu
\end{array}\right] \omega_{1}^{2},
\end{aligned}
$$

being $g=s / \omega_{1}$, and the force vector:

$$
\left[\begin{array}{l}
F_{1}(s) \\
F_{2}(s)
\end{array}\right]=\left[\begin{array}{c}
s 2 \zeta_{1} \omega_{1}+\omega_{1}^{2} \\
0
\end{array}\right] \bar{X}_{g}=\left[\begin{array}{c}
g 2 \zeta_{1}+1 \\
0
\end{array}\right] \bar{X}_{g} \omega_{1}^{2} .
$$

Then, the dynamic response of the structural system in terms of displacement, as a function of $s$, can be obtained just through an algebraic manipulation of Eq. (21):

$$
\mathbf{X}(s)=\mathbf{Z}(s)^{-1} \mathbf{F}(s)=\mathbf{H}(s) \mathbf{F}(s)
$$

where $\mathbf{H}(s)=\mathbf{Z}(s)^{-1}$ is the receptance matrix of the system:

$$
\begin{aligned}
\mathbf{H}(s) & =\left[\begin{array}{cc}
H_{11}(s) & H_{12}(s) \\
H_{21}(s) & H_{22}(s)
\end{array}\right]= \\
& =\frac{1}{D(s)}\left[\begin{array}{cc}
s^{2} \mu+s 2 \zeta_{2} \omega_{2} \mu+\omega_{2}^{2} \mu & s 2 \zeta_{2} \omega_{2} \mu+\omega_{2}^{2} \mu \\
s 2 \zeta_{2} \omega_{2} \mu+\omega_{2}^{2} \mu & s^{2}+s\left(2 \zeta_{1} \omega_{1}+2 \zeta_{2} \omega_{2} \mu\right)+\left(\omega_{1}^{2}+\omega_{2}^{2} \mu\right)
\end{array}\right]= \\
& =\frac{\omega_{1}^{2}}{D(s)}\left[\begin{array}{cc}
g^{2} \mu+g 2 f \zeta_{2} \mu+f^{2} \mu & g 2 f \zeta_{2} \mu+f^{2} \mu \\
g 2 f \zeta_{2} \mu+f^{2} \mu & g^{2}+g 2\left(\zeta_{1}+f \zeta_{2} \mu\right)+\left(1+f^{2} \mu\right)
\end{array}\right],
\end{aligned}
$$

where:

$$
\begin{aligned}
\frac{D(s)}{\omega_{1}^{4}} & =\frac{\operatorname{det}[\mathbf{Z}(s)]}{\omega_{1}^{4}}= \\
& =g^{4} \mu+g^{3} 2 \mu\left(\zeta_{1}+f \zeta_{2}(1+\mu)\right)+g^{2} \mu\left(1+f^{2}(1+\mu)+4 f \zeta_{1} \zeta_{2}\right)+ \\
& +g 2 f \mu\left(f \zeta_{1}+\zeta_{2}\right)+f^{2} \mu .
\end{aligned}
$$

Hence, one obtains:

$$
\begin{aligned}
& \frac{X_{1}(s)}{\bar{X}_{g}}=H_{11}(s) F_{1}(s)+H_{12}(s) F_{2}(s)=\frac{N_{1}(s)}{D(s)}, \\
& \frac{X_{2}(s)}{\bar{X}_{g}}=H_{21}(s) F_{1}(s)+H_{22}(s) F_{2}(s)=\frac{N_{2}(s)}{D(s)}
\end{aligned}
$$

where, in the present case:

$$
\begin{aligned}
& \frac{N_{1}(s)}{\omega_{1}^{4}}=g^{3} 2 \zeta_{1} \mu+g^{2} \mu\left(4 f \zeta_{1} \zeta_{2}+1\right)+g 2 f \mu\left(f \zeta_{1}+\zeta_{2}\right)+f^{2} \mu, \\
& \frac{N_{2}(s)}{\omega_{1}^{4}}=g^{2} 4 f \zeta_{1} \zeta_{2} \mu+g 2 f \mu\left(f \zeta_{1}+\zeta_{2}\right)+f^{2} \mu .
\end{aligned}
$$

The time response is finally obtained through the inverse Laplace transform of Eq. (27). However, even for a relatively simple system as that assumed in this study, the analytical expressions of this time response take too lengthy forms to be reported here. 


\subsection{Expected effects of the tuning variables}

In order to obtain first useful indications for the following TMD tuning process, a preliminary investigation on the expected trends of the structural displacement as a function of the tuning variables has been developed, providing the following main tuning guidelines.

The $\mathrm{H}_{2}$ norm (average response) of the primary structure displacement, represented in Fig. 3, exhibits a quite convex shape, which fact pushes this quantity as a potentially suitable objective function within an optimisation process devised for the TMD tuning. Besides this character, it was found that the range of frequency ratio $f$ including the minimum is a bit extended, meaning that also values not corresponding to the actual optimum one could still lead to an effective TMD. This important consideration holds true also for TMD damping ratio $\zeta_{2}$, and it appears constant also for different values of mass ratio $\mu$.

Figure 3

Hence, the features recovered from this preliminary analysis point out that even a limited variation of the TMD parameters, possibly associated to de-tuning [34], should not much jeopardise the effectiveness of the passive control device in the present impulse excitation context. Also, this flattens out the importance of the specific adopted tuning approach, if still effective in anyway localising the TMD parameters at around the global optimum.

\section{$3 \quad$ TMD tuning process}

The present TMD tuning process can be interpreted and managed by an appropriate optimisation procedure, where the objective (or cost) function is assumed to be a quantity representative of the dynamic response of the structure, either in the time or in the frequency domain, which is supposed to be minimised [36,37]:

$$
\min _{\mathbf{p}} \mathrm{J}(\mathbf{p}), \quad \mathbf{l}_{b} \leq \mathbf{p} \leq \mathbf{u}_{b},
$$

where $\mathbf{p}, J(\mathbf{p}), \mathbf{l}_{b}$ and $\mathbf{u}_{b}$ represent the optimisation variables, the objective function, the lower and the upper bounds on the optimisation variables, respectively. In the present context, following a typical approach adopted in the TMD literature $[4,6,10,14,19,20,34,35,48]$, only frequency ratio $f$ and TMD damping ratio $\zeta_{2}$ are taken as variables for a time-domain optimisation process, while mass ratio $\mu$ is assumed to be given from scratch (two-variable optimisation: $\mathbf{p}=\left[f ; \zeta_{2}\right]$ ), based on values that may be encountered in real applications.

This two-variable optimisation approach is motivated by two main reasons. First, the amount of added mass which composes the TMD is generally limited by a matter of practical design, i.e. excessive masses of the device could be counterproductive in terms of suitability and safety of the primary structure, especially if post-fitted by adding a TMD control device. The second issue, which is instead related to rather theoretical aspects, is pointed out by the optimisation process itself. Indeed, several previous tuning trials [36,37] have shown that, in case of a free mass ratio, this parameter tends to reach the upper limit that has been set, leading to a lower TMD damping ratio, so that to transfer the dynamic response entirely to the TMD. In this sense, a suitable upper limit to the design guidelines could be established within the optimisation process, which will likely correspond to the assumed value of $\mu$. In practice, TMD efficiency is expected to raise at increasing mass ratio, specifically in this pulse-like context, but the added TMD mass shall be bounded by feasible constraints. Despite this, such considerations are worthwhile to be explicitly mentioned, since some different studies have pushed to investigate even rather high values of mass ratios (e.g., in the present pulse-like context, up to 50\% [25]), and sometimes the 
mass ratio is still kept among the optimisation variables (e.g. [8], although likely systematically falling on the admitted upper-bound value or around it).

The characteristics of the considered objective function play a fundamental role for the real effectiveness of the optimisation process. Above all, despite that a relatively simple system has been here assumed, namely a 2DOF structure, the analytical expressions of its dynamic response quickly take a complex form, which leads to consider a numerical optimisation method as a suitable mean to develop the TMD tuning process.

Another important feature, within the tuning process, regards the choice of the optimisation algorithm. In this sense, despite that a large series of modern algorithms may assure even higher optimisation performances (see e.g. [20], and [8], with references quoted therein), a classical numerical method looks rather sufficient, and robust, for the solution of the present tuning problem. Indeed, the computational time required by the optimisation process has been noticed to be quite short, probably because of the simplicity of the structural system and the deterministic nature of the dynamic excitation. Particularly, a classical nonlinear gradient-based optimisation algorithm has been adopted, which is based on Sequential Quadratic Programming $(S Q P)$ and takes advantage of the fmincon function within MATLAB.

The ranges of parametric values of mass ratio $\mu$ and structural damping ratio $\zeta_{1}$ have been chosen similarly to those typically appearing in engineering applications (as expressed in MATLAB notation):

$$
\boldsymbol{\mu}=[0.0025: 0.0025: 0.1], \quad \boldsymbol{\zeta}_{1}=[0,0.01,0.02,0.03,0.05] .
$$

Namely, mass ratio $\mu$ ranges from $0.25 \%$ to $10 \%$, with steps of $0.25 \%$ and inherent damping ratio $\zeta_{1}$ takes values of 0 (no damping) and 1\%,2\%,3\%, $5 \%$, thus pushing to relatively high damping ratios in terms of challenging TMD effectiveness. Moreover, the values assumed for the tolerances and the maximum value of iterations and function evaluations were able to ensure both fast convergence and high level of accuracy. Further details on the implemented algorithm of TMD tuning are provided in [37].

In order to find out the best objective function for the tuning process, a preliminary investigation had been carried out on several response indices, which were actually the $\mathrm{H}_{\infty}$ (peak value) and the $\mathrm{H}_{2}$ (average value) norms of the primary structure displacement (see Section 2.2); recall also prodromal considerations at the end of Section 2.5. The results obtained from the optimisation trials provided the following useful indications. In general, the assumption of a $\mathrm{H}_{\infty}$ response quantity leads to undesired results, since frequency ratio $f$ takes values corresponding to the upper bound, while TMD damping ratio $\zeta_{2}$ displays abnormal decreasing trends, and the dynamic response appears not to take appreciable advantages from the presence of the soconceived TMD. In fact, the peak response is just a little reduced, while the overall response appears to be even increased. Actually, the norm which rendered the best results is the $\mathrm{H}_{2}$ norm of the considered response quantities, because of the regular trends of the optimum TMD parameters and of the remarkable efficiency in reducing the overall dynamic response. Hence, the $\mathrm{H}_{2}$ norm of the primary structure displacement has been eventually assumed as objective function within the final optimisation process.

\section{Optimum TMD parameters for impulse loading}

The optimum TMD parameters for impulse base displacement obtained by the tuning methodology above have been represented in Fig. 4. The main trends of the optimum TMD parameters substantially provide a decreasing frequency ratio $f^{\text {opt }}$ and an increasing TMD damping ratio $\zeta_{2}^{o p t}$ at increasing mass ratio $\mu$. In general, $f^{\text {opt }}$ only slightly decreases at increasing primary structure damping ratio $\zeta_{1}$, while $\zeta_{2}^{o p t}$ exhibits a general high level of insensitivity with respect 
to this parameter. Thus, a main message of insensitivity of optimum TMD parameters $f^{\text {opt }}, \zeta_{2}^{\text {opt }}$ for impulse base displacement on inherent structural damping ratio $\zeta_{1}$ globally appears from Figs. $4 \mathrm{a}$ and $4 \mathrm{~b}$, in the inspected window of reasonable mass ratios up to $10 \%$ (already a rather high value).

The TMD tuning based on classical Den Hartog formulae [10]

$$
f=\frac{1}{1+\mu}, \quad \zeta_{2}=\sqrt{\frac{3 \mu}{8(1+\mu)}}
$$

is also taken into account as a universal reference for comparison purposes (despite for being originally obtained for harmonic force and undamped primary structure) and provided values of $f$ and $\zeta_{2}$ respectively lower and higher than those achieved with the present impulse-based tuning method, including for the case of undamped primary structure $\left(\zeta_{1}=0\right)$, which indeed constitutes the reference in Den Hartog analysis. Thus, while an overshooting is recovered for $f^{\text {opt }}$, an undershooting is recorded for $\zeta_{2}^{o p t}$, with respect to classical Den Hartog reference, which was considered for harmonic force loading.

A further comparison of the presented impulse-tuned optimum parameters with respect to those obtainable in case of different external excitations is now proposed. In particular, the tuning formulae for the cases of harmonic and white noise excitations, either applied as force on the primary structure or as base acceleration, taken from previous work [38] (separately reported in Appendix A), have been as well considered for this confrontation analysis on the optimum TMD parameters.

The relevant results are represented in Fig. 5, and will be discussed in the following. First, the main trends are confirmed, i.e. for all the considered loading cases, frequency ratio $f$ decreases and TMD damping ratio $\zeta_{2}$ increases at increasing mass ratio $\mu$. The trends of $f$ are a bit different from each other, and in this sense the trend related to impulse loading is placed halfway with respect to the others, quite similarly to the values of $f$ for the case of excitations applied as base acceleration (both harmonic and white noise loadings). However, this fact appears not to be related to the present unit impulse applied as base displacement, since almost the same parameters have been obtained from further separate tests in the case of unit impulse force on the primary structure, which are not here reported.

On the other hand, the different trends of $\zeta_{2}$ look narrower, with two main streams defined by the harmonic and white noise excitations, respectively [38]. In this context, the parameters obtained for the case of unit impulse base displacement closely follow the trend outlined for the white noise excitations. In general, the above discussed results seem to support the concept of a sort of unified tuning, i.e. a tuning of TMD parameters that could reasonably approximate the optimum tuning of the three different considered excitation cases.

Figures 4-5

\section{Time response reduction}

\subsection{Unit impulse response}

The effectiveness of the proposed impulse-tuned TMD, in terms of reduction of time primary structure response $x_{1}(t)$, is the subject of the present subsection, where the unit impulse base displacement is still considered as acting dynamic excitation, coherently with the previous analytical response and numerical tuning analysis.

In Table 1, the percentage magnitudes of analytical response reduction, in terms of $\left\|x_{1}(t)\right\|_{\infty}$ and $\left\|x_{1}(t)\right\|_{2}$ norms have been gathered, which, for the SDOF primary structure alone, are 
represented by Eqs. (13) and (14) respectively (Section 2.2), for values $\boldsymbol{\zeta}_{\mathbf{1}}=[0,0.03,0.05]$ and $\boldsymbol{\mu}=[0.02,0.05,0.1]$. One may observe that the peak response abatement is rather small, also for significant values (from an engineering application point of view) of mass ratio $\mu$, and it exhibits a high insensitivity on structural damping ratio $\zeta_{1}$. On the other hand, the overall response decrease is characterised by remarkable values, especially for high values of $\mu$ and low values of $\zeta_{1}$, and it results to be quite sensitive to these structural parameters, i.e. $\Delta\left\|x_{1}(t)\right\|_{2}$ increases at increasing $\mu$ and decreasing $\zeta_{1}$. Similar results are also recorded from a tuning based on classical Den Hartog reference, Eq. (31), as further reported in Table 2. Despite for the optimum TMD parameters being different (see earlier discussion in Section 4 and specifically trends depicted in Fig. 4), the final vibration mitigation performance turns out to be rather similar, in this context, as already conjectured at the end of Section 2.3 (see Fig. 3).

\section{Tables 1-2}

These considerations on the overall response reduction are further confirmed in plot form by the inspection of Fig. 6a on the left. The percentage reduction of the $\mathrm{H}_{2}$ norm of the primary structure displacement, here adopted as a suitable TMD performance index, shows similar trend shapes as a function of $\mu$, for all the different considered values of $\zeta_{1}$, but the reduction values turn out quite different, i.e. one may note that the lower $\zeta_{1}$, the higher the response reduction, as it may be expected from previous TMD tuning experience [37-40]. Again, quite similar results are also depicted in Fig. 6b on the right, for Den Hartog tuning.

From the further time-history responses in Figs. 7a and 8a on the left, it can be observed that the increase of mass ratio $\mu$ turns out in a remarkable increase of TMD efficiency, especially after the very beginning of the dynamic response (i.e. the first two/three peaks of oscillation). Further investigations pointed out that such a fact is more evident for the lower values of $\zeta_{1}$. However, the amplitude of primary structure displacement $x_{1}(t)$ tends to zero after more or less the same duration (e.g. 6-8 seconds, for the considered primary structure), independently on the assumed value of $\mu$. Similar time-history results are again recorded in Figs. 7b and 8b on the right, for Den Hartog tuning, definitively showing this to constitute a consistent and feasible way of tuning to achieve vibration mitigation also for this impulse-like base excitation context, despite for being originally conceived for an undamped primary structure under harmonic force.

Despite for the global considerable TMD efficiency, the maximum response, which in case of impulsive loading for a system initially at rest occurs at the first peak of dynamic response, does not seem to be substantially affected by the insertion of a passive TMD (at least for the considered reasonable contained values of mass ratio $\mu$ ). This may suggest the further application of a hybrid controller, based on an underlying setting relying on the present passive TMD tuning procedure, apt to improve the achievable control performance also in terms of $\mathrm{H}_{\infty}$ norm.

Figures 6-8

\subsection{Other post-tuning pulse-like excitation responses}

In order to assess, and possibly to extend, the validity of the proposed TMD tuning procedure for unit impulse base displacement excitation, the so-conceived pulse-tuned optimum TMD has been tested for further four post-tuning pulse-like excitations, representative of real sudden actions with a variable duration, namely versed-sine pulse, linear ramp, cubic ramp and unit step (whose analytical statement is separately reported in Appendix B).

The amplitude of the signal has been assigned so that to satisfy the condition of unitary impulse, as for a Dirac delta function (beginning of Section 2.1), i.e. so that to ensure a common 
unitary character of the pulse loading and to achieve similar amplitudes for the first peak of displacement response, leading to a base displacement constant impulse amplitude of $\bar{X}_{g}=0.01 \mathrm{~m} \mathrm{~s}$, $\bar{X}_{g}=0.008 \mathrm{~m} \mathrm{~s}, \bar{X}_{g}=0.007 \mathrm{~m} \mathrm{~s}, \bar{X}_{g}=0.01 \mathrm{~ms}$, for the versed-sine pulse, the linear ramp, the cubic ramp and the unit step case, respectively. Pulse-like excitations are investigated for different values of excitation period $T_{p}$, with respect to natural period $T_{1}$, in order to enquire the effect of different excitation durations for the pulse-like profile.

The post-tuning trials, with given values of mass ratio $\boldsymbol{\mu}=[0.02,0.05]$ and structural damping ratio $\boldsymbol{\zeta}_{1}=[0,0.03]$, in order to appreciate TMD effectiveness at a widening spreading of them, have been carried out by considering three different preceding tuning methods: (a) Impulsetuned TMD for the case of base displacement (Section 4), which is the method that has been previously described and newly here proposed; (b) Classical reference Den Hartog tuning [10], originally outlined from an undamped primary structure subjected to harmonic force, Eq. (31); (c) Assigned TMD parameters, namely $f=1$ and $\zeta_{2}=0.15$, as typical reference values for practical design purposes. From the results obtained at a preliminary stage in Section 2.3, it appears that a suitable choice of the TMD parameters may contemplate slightly lower values than those indicated above. However, this set of TMD parameters may be considered as adequately representative of a possible real TMD. Moreover, the assumption of a resonant TMD $(f=1)$ has been proposed and validated in relevant literature works [45, 46].

Tables 3-6 display the achieved percentage response reductions, evaluated as average values of those achieved for the four pulse-like excitations, for different values of primary structure damping ratio $\zeta_{1}\left(\zeta_{1}=0\right.$ in Tables 3 and $4 ; \zeta_{1}=0.03$ in Tables 5 and 6$)$ and mass ratio $\mu$ $(\mu=0.02$ in Tables 3 and $5 ; \mu=0.05$ in Tables 4 and 6$)$, and for different amounts of pulse excitation frequency, which cover the range from a situation of sudden loading $\left(T_{p}=0.1 T_{1}\right)$, to a case where the loading period is close to the structural one $\left(T_{p}=0.8 T_{1}\right)$.

\section{Tables 3-6}

The representation of an average value is motivated by the fact that, especially for actual pulse loading $\left(T_{p}=0.1 T_{1}\right)$, the obtained values of response reduction are almost the same for the different excitations. This similarity of results tends instead to disappear when the excitation period increases $\left(T_{p}=0.8 T_{1}\right)$, and the shape of the input signal affects a bit more the TMD efficiency. In particular, this holds true for the case of unit step pulse.

Hence, from these results, it appears that for shock loading, i.e. in case of short-duration actions, the nature of the impulsive excitation does not significantly affect the TMD performance, while this feature gradually influences the response reduction at an increasing duration of the external dynamic action.

In general, the peak response $\left(\mathrm{H}_{\infty}\right.$ norm $)$ is just slightly reduced, while the overall response $\left(\mathrm{H}_{2}\right.$ norm $)$ results to be quite lowered; such a situation holds true even when the period of the excitation gets closer to that of the primary structure, i.e. when the sudden character of the excitation is actually missing. Also, the TMD performance decreases at decreasing pulse period, especially when the loading exhibits a period close to that of the system, losing therefore the character of a sudden dynamic action.

The differences of performance between the various tuning methods appear quite small but, on the whole, one could notice that the results coming from the proposed impulse tuning are just slightly better than those obtained from Den Hartog tuning, which is indeed confirmed as a valid benchmark reference, even in the damped case, while the case of assigned parameters provides a slightly higher reduction of peak response, even if not really significant (lower than $2.5 \%$ ). As expected, the achieved TMD benefit increases at increasing mass ratio $\mu$, especially as referred to the overall response reduction, which switches from $52-62 \%$ for $\mu=0.02$ to $63-70 \%$ for $\mu=0.05$, when $\zeta_{1}=0$, and from $20-28 \%$ for $\mu=0.02$ to $29-37 \%$ for $\mu=0.05$, when $\zeta_{1}=0.03$. 
Also, TMD effectiveness is confirmed to be much lower for the higher inherent damping ratio $\left(\zeta_{1}=0.03\right)$, than for the undamped case $\left(\zeta_{1}=0\right)$, despite for giving anyway visible response reductions, which indeed motivates the insertion of the TMD device, disregarding for the specific adopted tuning method.

\subsection{Application case study: seismic response reduction}

The efficiency of the proposed pulse-tuned TMD has been also investigated for the post-tuning case of non-stationary seismic excitation. Indeed, to enhance the implications and potential applications of the proposed impulse tuning concept, the important seismic scenario of earthquake response is further considered. The passive TMD is first tuned for impulse base displacement, following the procedure outlined above (Section 4). Then, its effectiveness is evaluated in terms of post-tuning achieved seismic response reduction of the primary structure.

Due to the unique nature of each seismic event, several earthquakes have been considered within this investigation, acting as base acceleration on the structural system. Since the analytical Laplace transform can not be obtained for generic seismic input, here the time response has been computed through a classical numerical direct integration technique of the equations of motion in the time domain, by means of Newmark average acceleration method.

An extract of the outcomes of this seismic numerical investigation, based on four instances of earthquake recordings (Imperial Valley 1940; Loma Prieta 1989; Kobe, 1995; L'Aquila, 2009), has been here reported by assuming the following two structural instances in terms of values of underlying structural parameters: (a) mass ratio $\mu=0.02$ and structural damping ratio $\zeta_{1}=0 ;(\mathbf{b})$ mass ratio $\mu=0.05$ and structural damping ratio $\zeta_{1}=0.03$. These two choices go as follows: first case (a) refers to a possible realistic case of TMD insertion, with reasonable low added TMD mass on a lightly damped (undamped) structure, a typical scenario of expected effective TMD application; second case (b) points out to a limit investigation case in which, on one hand, a rather high value of mass ratio has been assumed, in order to inspect how seismic response mitigation may be pushed up by a TMD insertion, on the other hand, an already challenging value of structural damping ratio has been considered, since a lower TMD efficiency shall be recorded at increasing amount of inherent structural damping. The obtained numerical percentage reductions in terms of $\left\|x_{1}(t)\right\|_{\infty}$ and $\left\|x_{1}(t)\right\|_{2}$ norms are listed in Tables 7-8, again with comparison to classical Den Hartog tuning, while the related time histories are shown in Figs. 9-10.

\section{Tables 7-8}

Figures $9-10$

In general, the peak response reduction appears to be smaller, i.e. nearly one third, than for the overall displacement decrease, except for one earthquake case (Loma Prieta). The ratio between the two indices is apparently quite variable and it seems that a clear relationship could not be outlined on the considered earthquake set. This is somehow expected, for the different characteristics of the seismic signals, especially in terms of signal power and frequency content.

On the other hand, as a general important consideration, the effectiveness of the passive TMD, earlier optimised for a unit impulse base displacement excitation, turns out to be quite significant also for the case of earthquake loading. Indeed, average values of $15 \%$ of $\left\|x_{1}(t)\right\|_{\infty}$ and $30 \%$ of $\left\|x_{1}(t)\right\|_{2}$ unwanted response are suppressed by the addition of the TMD, for the relatively high value of $\zeta_{1}=0.03$, which is a performance that provides a noticeable benefit to the primary structure and therefore supports the adoption of this control device in the seismic engineering context, even if optimised for a different source dynamic excitation (impulse loading). 
Comparison to Den Hartog tuning shows rather similar results, which confirms this to constitute a consolidated general tuning concept, also within this seismic field.

The time histories shown in Figs. 9-10 also allow to add further relevant considerations. First, the small effect of the TMD in reducing the sudden peaks of response is confirmed, as it can be especially pointed out from the plots related to the Imperial Valley, Kobe and L'Aquila earthquakes. However, after these excitation peaks, the TMD starts to properly operate within the dynamic response, and a remarkable reduction of subsequent oscillations is generally attained, despite for the average, reasonable value of assumed inherent structural damping already challenging the TMD effectiveness $\zeta_{1}=0.03$; see the effect of $\zeta_{1}$ on the TMD performance in Table 1). Moreover, such a positive behaviour usually holds true until the end of the seismic excitation, unless if a new peak in the input time history may come up.

\section{Resuming technical discussion on the obtained results}

This study concerned the optimisation of a passive Tuned Mass Damper device for linear mechanical systems subjected to transient loading, in the form of unit impulse excitation, acting as base displacement, assuming as primary structure a SDOF structural system (which may be emblematic also of a MDOF system controlled on a single mode of vibration). The dynamic response of such a structural system has been analytically obtained, through a pair of Laplace transforms, and then adopted for numerical TMD tuning purposes. This work also forms a theoretical background for a possible further upgrade of the passive TMD device to a smarter hybrid version, whose features are complementary outlined in [36].

The impulse-tuned TMD calibration process involved the development of a numerical optimisation procedure, carried out by means of a nonlinear algorithm based on Sequential Quadratic Programming (SQP). In a preliminary round of analysis, different response quantities have been assumed to characterise the objective function, so that to find out the best control function in terms of TMD performance and stability of the whole tuning process. The evaluation of the objective function, i.e. of its most pertinent norm, has been investigated as well. The tests and related outcomes revealed that the best choice as cost function appeared to be the $\mathrm{H}_{2}$ norm of primary structure displacement $x_{1}(t)$, namely an average quantity over the whole window of transient analysis. Other response quantities could represent valid alternatives, the key point being their evaluation as $\mathrm{H}_{2}$ norm, since other norms rendered rather unsatisfactory results, from both the point of view of optimum TMD parameters (main problems in this sense could be either constituted by uncertainties on the optimum values or the reaching of the given bounds) and effective performance of the control device. The optimum TMD parameters exhibit trends that are quite similar to those obtainable for other benchmark excitations, such as harmonic or white noise $[10,38]$. This feature turns out to be quite useful in view of a possible comprehensive tuning of passive TMD devices.

Several post-tuning numerical tests on the structural time response have been carried out after the impulse-based TMD tuning process, in order to quantify the amount of potential TMD effectiveness within the transient loading regime. Firstly, the same excitation assumed in the optimisation process, i.e. a unit impulse base displacement, has been considered as dynamic loading. The results at that stage pointed out a remarkable efficiency of the so-conceived optimum TMD, since it allowed to reduce the overall dynamic response of the primary structure by a significant amount, for instance in the order of about $30 \%$, for mass ratio $\mu=0.02$ and structural damping ratio $\zeta_{1}=0.03$, and also for $\mu=0.05, \zeta_{1}=0.05$. On the other hand, it appears that the passive TMD device is not effective in reducing as well the peak response, likely because of its inertial behaviour, despite for the small added mass, which does not allow to promptly react to a sudden excitation, as it is for the case of unit impulse here considered. 
Indeed, negligible percentage reductions have been obtained in terms of the $\mathrm{H}_{\infty}$ norm of $x_{1}(t)$, at least for the reasonable, realistic values of mass ratio considered within the study. This constitutes the main motivation for a possible further improvement by the insertion of a hybrid controller, apt to control the peak response, too. Despite, good response reductions in terms of $\mathrm{H}_{2}$ norm (average response) truly motivate the a priori adoption of such a pulse-tuned (even passive) TMD, made anyway available even if the active controller may be found turned off.

In a second session of tests, four additional post-tuning pulse-like excitations have been considered, so as to enquire the potential effectiveness of the control device for generic sudden dynamic actions of a variable duration, also through a comparison with other tuning methods, namely by classical Den Hartog tuning formulae or by the assignment of TMD parameters given from scratch, close to those usually adopted for real applications, due to design or availability constraints. The so-obtained results, quite similar for the different pulse-like excitations, mainly pointed out an overall response that is even more reduced than for the peak displacement, confirming therefore what obtained for the previous ideal impulse excitation case. As a main general remark, the TMD is acknowledged to produce an abatement of at least $1 / 4$ (which becomes $1 / 3$ for the higher values considered for the mass ratio) of the total response, with consequent significant benefit supplied to the structural system, despite for the reasonable, relatively high value of the assumed structural damping ratio, in the order of $3 \%$, which already much challenges the achievable level of TMD effectiveness (TMDs are indeed expected to produce significant benefits for undamped or very lightly-damped primary structures). Such a fact supports the assumption of passive TMDs for impulsive response reduction, since it appears that, in principle, such devices shall provide a remarkable benefit to the host structure, especially all along the whole window of transient dynamic response. Moreover, being in a sense not so sensitive to the specific adopted tuning procedure (see discussion at the end of Section 2.3), with a similar effectiveness displayed through classical Den Hartog tuning, the response to pulse-like loading with TMD should also become rather independent of possible phenomena of de-tuning, which may occur as a consequence of modifications in the structural properties, and of different possible tunings.

In a third assessment investigation, the effectiveness of the pulse-tuned passive TMD has been explored within the seismic engineering range, by considering the primary structure response reduction under different earthquake instances. This has demonstrated that the TMD tuning based on impulse excitation shall provide an effective way of tuning the passive TMD device toward possible seismic isolation and control for a priori unknown seismic input. Moreover, in terms of achieved seismic response reduction, the above positive features have been also revealed for the seismic excitation case, where a visible level of average response reduction at about $30 \%$ has been recorded, even for the (relatively high but reasonable) value assumed for the structural damping ratio at $3 \%$. Less effect on the peak response reduction is again noticed (for the examined contained values of mass ratio).

The following main remarks out of the present analytical/numerical theoretical investigation may be itemizely resumed as follows:

- $\mathrm{H}_{2}$-norm (i.e. average response) optimisation of the TMD tuning concept has revealed to be robust and effective in estimating the TMD tuning parameters under impulse excitation. Associated to that, the higher TMD performance under impulse loading has been recorded on the average response, rather than on the peak response.

- The post-addition of an active controller, to make the passive TMD to become a hybrid device, may further help in controlling the peak response, too. Like that, the classical (passive) device may be alerted with no active control available or turned off and mitigate 
the average response following a transient impulse excitation. With the active control turned on there appears the possibility to force the control of the peak response, too. However, the classical passive configuration shall provide a first basic reliable and effective protection for the after-pulse vibration.

- Trends of the obtained optimum TMD parameters are similar to those obtained for reference excitations, with very little effect of changes in the structural damping ratio. With respect to classical Den Hartog tuning, frequency ratio overshoots, while TMD damping ratio undershoots, while final results in terms of response mitigation turn out rather similar, with the present tuning generally leading to slightly better abatements. Since achievements are anyway good, this supports the option of TMD insertion for this transient loading context, disregarding for the specific TMD tuning concept being selected.

- Post-tuning tests, including pulse-like and earthquake excitations, have confirmed the response control trends experienced for impulse tuning, in terms of considerable abatement of the average structural response, increasing at raising mass ratio and lowering structural damping ratio.

\section{Conclusion}

In conclusion, the main results of this research study outline several crucial indications on the optimum design of passive Tuned Mass Dampers for the control of transient impulse responses of structural systems. In particular, the basic addition of a passive TMD control device to a structural system could significantly abate its transient impulsive response, with a remarkable benefit in terms of serviceability and safety, specifically on the average response indices evaluated all along the whole transient window of analysis. The reduction of the peak response looks instead less effective (for contained, reasonable values of added TMD mass) and may require the further addition of a smarter active controller to the passive control device, towards achieving an even more effective hybrid TMD, to be turned on for mitigating the peak response, too.

The overall TMD tuning and effectiveness analysis here developed and presented for impulsive excitation claims for a passive TMD to be anyway inserted, disregarding for the specificities of the adopted tuning method, provided that the TMD parameters shall take values within typical reasonable ranges for practical applications. This supports the general concept that, for transient, pulse-like loading, or earthquake excitation, the presence of a passive TMD shall ensure a reliable basic protection against unwanted transient vibration, with a low sensitivity on the specific adopted TMD tuning method and thus on possible de-tuning effects.

\section{Acknowledgements}

The Authors would like to acknowledge public research funding from "Fondi di Ricerca d'Ateneo ex 60\%", at the University of Bergamo, Department of Engineering and Applied Sciences (Dalmine), a former ministerial doctoral grant at the MITIMM Doctoral School of the same and research project funding "FYRE - Fostering Young REsearchers" supported by Fondazione Cariplo at the University of Bergamo, which allowed for exchange research at the Institute of Sound and Vibration Research (ISVR), University of Southampton. 


\section{References}

[1] Abé, M., Igusa, T., 1996, Semi-active dynamic vibration absorbers for controlling transient response, Journal of Sound and Vibration, 198(5):547-569.

[2] Adam, C., Furtmüller, T., 2010, Seismic Performance of Tuned Mass Dampers, in: H. Irschik, M. Krommer, K. Watanabe, T. Furukawa (Eds.), Mechanics and Model-Based Control of Smart Materials and Structures, Springer-Verlag, 1st ed., pp. 11-18.

[3] Aly, A.M., 2014, Vibration control of high-rise buildings for wind: a robust passive and active tuned mass damper, Smart Structures and Systems, 13(3):473-500.

[4] Asami, T., Nishihara, O., Baz, A.M., 2002, Analytical solutions to $\mathrm{H}_{\infty}$ and $\mathrm{H}_{2}$ optimization of dynamic vibration absorber attached to damped linear systems, Journal of Vibration and Acoustics, ASME, 124(2):284-295.

[5] Bakre, S.V., Jangid, R.S., 2004, Optimum multiple tuned mass dampers for base-excited damped main system, International Journal of Structural Stability and Dynamics, 4(4):527542.

[6] Bakre, S.V., Jangid, R.S., 2006, Optimum parameters of tuned mass damper for damped main system, Structural Control and Health Monitoring, 14(3):448-470.

[7] Bandivadekar, T.P., Jangid, R.S., 2013, Optimization of multiple tuned mass dampers for vibration control of system under external excitation, Journal of Vibration and Control, 19(12):1854-1871.

[8] Bekdaş, G., Nigdeli, S.M., 2017, Metaheuristic based optimization of tuned mass dampers under earthquake excitation by considering soil-structure interaction, Soil Dynamics and Earthquake Engineering, 92(January 2017):443-461.

[9] Chen, G., Wu, J., 2003, Experimental study on multiple tuned mass dampers to reduce seismic responses of a three-storey building structure, Earthquake Engineering and Structural Dynamics, 32(5):793-810.

[10] Den Hartog, J.P., 1956, Mechanical Vibrations, McGraw-Hill, 4th ed.

[11] Desu, N.B., Dutta, A., Deb, S.K., 2007, Optimal assessment and location of tuned mass dampers for seismic response control of a plan-asymmetrical building, Structural Engineering and Mechanics, 26(4):459-477.

[12] Farshidianfar, A., Soheili, S., 2013, Ant colony optimization of tuned mass dampers for earthquake oscillations of high-rise structures including soil-structure interaction, Soil Dynamics and Earthquake Engineering, 51(August 2013):14-22.

[13] Ghahari, S.F., Abazarsa, F., Taciroglu, E., 2017, Blind modal identification of nonclassically damped structures under non-stationary excitations Structural Control and Health Monitoring, 24(6):1-21, June 2017, Article number e1925.

[14] Ioi, T., Ikeda, K., 1978, On the dynamic vibration damped absorber of the vibration system, Bulletin of the Japanese Society of Mechanical Engineering, 21(151):64-71.

[15] Jabary, R.N., Madabhushi, G.S.P., 2015, Tuned mass damper effects on the response of multi-storied structures observed in geotechnical centrifuge tests, Soil Dynamics and Earthquake Engineering, 77(October 2015):373-380. 
[16] Jabary, R.N., Madabhushi, G.S.P., 2017, Tuned mass damper positioning effects on the seismic response of a soil-MDOF-structure system, Journal of Earthquake Engineering, 31 January 2017:1-22.

[17] Jabary, R.N., Madabhushi, G.S.P., 2017, Structure-soil-structure interaction effects on structures retrofitted with tuned mass dampers, Soil Dynamics and Earthquake Engineering, 100(September 2017):301-315.

[18] Jang, S.-J., Brennan, M.J., Rustighi, E., 2010, Comparing the performance of optimally tuned dynamic vibration absorbers with very large or very small moment of inertia, Journal of Vibration and Acoustics, ASME, 132(3):1-4.

[19] Krenk, S., Høgsberg, J., 2008, Tuned mass absorbers on damped structures under random load, Probabilistic Engineering Mechanics, 23(4):408-415.

[20] Leung, A.Y.T., Zhang, H., 2009, Particle Swarm Optimization of tuned mass dampers, Engineering Structures, 31(3):715-728.

[21] Li, S., Tang, J., 2017, On Vibration Suppression and Energy Dissipation Using Tuned Mass Particle Damper, Journal of Vibration and Acoustics, ASME, 139(1):011008-1-10.

[22] Liu, Y., Lin, C.-C., Parker, J., Zuo, L., 2016, Exact H2 Optimal Tuning and Experimental Verification of Energy-Harvesting Series Electromagnetic Tuned-Mass Dampers, Journal of Vibration and Acoustics, ASME, 138(6):061003-1-12.

[23] Marano, G.C., Greco, R., 2011, Optimization criteria for tuned mass dampers for structural vibration control under stochastic excitation, Journal of Vibration and Control, 17(5):679688.

[24] Matta, E., 2011, Performance of tuned mass dampers against near-field earthquakes, Structural Engineering and Mechanics, 39(5):621-642.

[25] Matta, E., 2013, Effectiveness of tuned mass dampers against ground motion pulses, Journal of Structural Engineering, ASCE, 139(2):188-198.

[26] Miranda, J.C., 2016, Discussion of system intrinsic parameters of tuned mass dampers used for seismic response reduction, Structural Control and Health Monitoring, 23(2):349-368.

[27] Morga, M., Marano, G.C., 2014, Optimization criteria of TMD to reduce vibrations generated by the wind in a slender structure, Journal of Vibration and Control, 20(16):2404-2416.

[28] Parulekar, Y.M., Reddy, G.R., 2009, Passive response control systems for seismic response reduction: A state-of-the-art review, International Journal of Structural Stability and Dynamics, 9(1):151-177.

[29] Pioldi, F., Ferrari, R., Rizzi, E., 2015, Output-only modal dynamic identification of frames by a refined FDD algorithm at seismic input and high damping, Mechanical Systems and Signal Processing, 68-69(February 2016):265-291, doi:10.1016/j.ymssp.2015.07.004.

[30] Pioldi, F., Ferrari, R., Rizzi, E., 2015, Earthquake structural modal estimates of multistorey frames by a refined Frequency Domain Decomposition algorithm, Journal of Vibration and Control, 23(13):2037-2063, doi:10.1177/1077546315608557. 
[31] Pioldi, F., Ferrari, R., Rizzi, E., 2017, Seismic FDD modal identification and monitoring of building properties from real strong-motion structural response signals, Structural Control and Health Monitoring, Early View: 9 February 2017, 24(11):1-20, e1982, November 2017, doi:10.1002/stc.1982.

[32] Pioldi, F., Salvi, J., Rizzi, E., 2016, Refined FDD modal dynamic identification from earthquake responses with Soil-Structure Interaction, International Journal of Mechanical Sciences, 127(July 2017):47-61, doi:10.1016/j.ijmecsci.2016.10.032.

[33] Quaranta, G., Mollaioli, F., Monti, G., 2016, Effectiveness of design procedures for linear TMD installed on inelastic structures under pulse-like ground motion, Earthquakes and Structures, 10(1):239-260.

[34] Rana, R., Soong, T.T., 1998, Parametric study and simplified design of tuned mass dampers, Engineering Structures, 20(3):193-204.

[35] Randall, S.E., Halsted, D.M., Taylor, D.L., 1981, Optimum vibration absorbers for linear damped systems, Journal of Mechanical Design, ASME, 103(4):908-913.

[36] Salvi, J., Rizzi, E., Rustighi, E., Ferguson, N.S., 2015, On the optimisation of a hybrid Tuned Mass Damper for impulse loading, Smart Materials and Structures, doi:10.1088/0964-1726/24/8/085010, 24(8)(2015)085010, 15 pp.

[37] Salvi, J., Rizzi, E., 2015, Optimum tuning of Tuned Mass Dampers for frame structures under earthquake excitation, Structural Control and Health Monitoring, doi:10.1002/stc.1710, $22(4): 707-715$.

[38] Salvi, J., Rizzi, E., 2016, Closed-form optimum tuning formulas for passive Tuned Mass Dampers under benchmark excitations, Smart Structures and Systems, doi:10.12989/sss.2016.17.2.231, 17(2):231-256.

[39] Salvi, J., Rizzi, E., 2017, Optimum earthquake-tuned TMDs: Seismic performance and new design concept of balance of split effective modal masses, Soil Dynamics and Earthquake Engineering, doi:10.1016/j.soildyn.2017.05.029, 101(October 2017):67-80.

[40] Salvi, J., Pioldi, F., Rizzi, E., 2016, Optimum Tuned Mass Dampers under seismic SoilStructure Interaction, Submitted for publication, revised version.

[41] Setareh, M., 2001, Use of semi-active mass dampers for vibration control of force-excited structures, Structural Engineering and Mechanics, 11(4):341-356.

[42] Sun, C., Nagarajaiah, S., Dick, A.J., 2014, Family of smart tuned mass dampers with variable frequency under harmonic excitations and ground motions: closed-form evaluation, Smart Structures and Systems, 13(2):319-341.

[43] Tigli, O.F., 2012, Optimum vibration absorber (tuned mass damper) design for linear damped systems subjected to random loads, Journal of Sound and Vibration, 331(13):30353049 .

[44] Tributsch, A., Adam, C., 2012, Evaluation and analytical approximation of Tuned Mass Damper performance in an earthquake environment, Smart Structures and Systems, 10(2):155-179.

[45] Villaverde, R., 1993, Reduction in seismic response with heavily-damped vibration absobers, Earthquake Engineering and Structural Dynamics, 13(1):33-42. 
[46] Villaverde, R., Koyama, L.A., 1993, Damped resonant appendages to increase inherent damping in buildings, Earthquake Engineering and Structural Dynamics, 22(6):491-507.

[47] Wang, J.-F., Lin, C.-C., 2015, Extracting parameters of TMD and primary structure from the combined system responses, Smart Structures and Systems, 16(5):937-960.

[48] Warburton, G.B., 1982, Optimum absorber parameters for various combinations of response and excitation parameters, Earthquake Engineering and Structural Dynamics, 10(3):381401.

[49] Wu, Q., Zhao, X., Zheng, R., 2016, Experimental Study on a Tuned-Mass Damper of Offshore for Vibration Reduction, Journal of Physics: Conference Series, MOVIC2016 \& RASD2016, IOP Publishing, 744(2016):012045(1-9).

[50] Xiang, P., Nishitani, A., 2015, Optimum design and application of non-traditional tuned mass damper toward seismic response control with experimental test verification, Earthquake Engineering and Structural Dynamics, 44(13):2199-2220.

[51] Zilletti, M., Elliott, S.J., Rustighi, E., 2012, Optimisation of dynamic vibration absorbers to minimise kinetic energy and maximise internal power dissipation, Journal of Sound and Vibration, 331(18):4093-4100.

\section{Appendix A. Optimum TMD tuning formulae for benchmark excitations}

In Table A.1, the optimum TMD tuning formulae from [38] adopted in Section 4 are reported.

Table A.1

\section{Appendix B. Pulse-like excitations}

A comprehensive scheme of pulse-like excitations adopted in Section 5.2, including formulation in both time and Laplace domains, is displayed in Fig. B.1.

Figure B.1 


\section{List of Tables}

1 Percentage reduction of $H_{\infty}$ and $H_{2}$ norms of primary structure displacement $x_{1}(t)$ as a function of different values of mass ratio $\mu$ and structural damping ratio $\zeta_{1}$, achieved with the proposed tuning method. . . . . . . . . . . . . .

2 Percentage reduction of $H_{\infty}$ and $H_{2}$ norms of primary structure displacement $x_{1}(t)$ as a function of different values of mass ratio $\mu$ and structural damping ratio $\zeta_{1}$, obtained with Den Hartog tuning method [10] . . . . . . . . . . . . . . . . .

3 Average percentage reduction of $H_{\infty}$ and $H_{2}$ norms of primary structure displacement $x_{1}(t)$, with $\mu=0.02, \zeta_{1}=0$, for the four considered pulse-like excitations (Appendix D) . . . . . . . . . . . . . . . . . . .

4 Average percentage reduction of $H_{\infty}$ and $H_{2}$ norms of primary structure displacement $x_{1}(t)$, with $\mu=0.05, \zeta_{1}=0$, for the four considered pulse-like excitations (Appendix B) . . . . . . . . . . . . . . . . . .

5 Average percentage reduction of $H_{\infty}$ and $H_{2}$ norms of primary structure displacement $x_{1}(t)$, with $\mu=0.02, \zeta_{1}=0.03$, for the four considered pulse-like excitations (Appendix B). . . . . . . . . . . . . . . . .

6 Average percentage reduction of $H_{\infty}$ and $H_{2}$ norms of primary structure displacement $x_{1}(t)$, with $\mu=0.05, \zeta_{1}=0.03$, for the four considered pulse-like excitations (Appendix B). . . . . . . . . . . . . . . . . .

7 Percentage reduction of $H_{\infty}$ and $H_{2}$ norms of primary structure displacement $x_{1}(t)$, with $\mu=0.02, \zeta_{1}=0$, for four different earthquake signals, with passive TMD optimised for unit impulse base displacement. . . . . . . . . . . . . . . .

8 Percentage reduction of $H_{\infty}$ and $H_{2}$ norms of primary structure displacement $x_{1}(t)$, with $\mu=0.05, \zeta_{1}=0.03$, for four different earthquake signals, with passive TMD optimised for unit impulse base displacement. . . . . . . . . . . .

A.1 Tuning formulae for the optimum TMD parameters as outlined in [38] for different dynamic excitations. . . . . . . . . . . . . . . . . . . 


\section{List of Figures}

$1 \quad$ Structural parameters and absolute dynamic degrees of freedom of a SDOF linear primary structure (index 1), subjected to generic base displacement $x_{q}(t) . .$. .

2 Structural parameters and absolute dynamic degrees of freedom of a 2DOF linear mechanical system comprised of a SDOF primary structure (index 1) equipped with an added TMD (index 2), subjected to generic base displacement $x_{g}(t)$. . . .

$3 \mathrm{H}_{2}$ norm of the primary structure displacement as a function of tuning variables $f, \zeta_{2}$, for $\zeta_{1}=0.03$ and for different values of mass ratio $\mu \ldots \ldots . \ldots \ldots$

4 Optimum TMD parameters at variable mass ratio $\mu$ for different values of structural damping ratio $\zeta_{1}$ : (a) frequency ratio fopt; (b) TMD damping ratio $\zeta_{2}^{o p t}$. . .

5 Optimum TMD parameters at variable mass ratio $\mu$, with structural damping ratio $\zeta_{1}=0.03$, for different dynamic excitations: (a) frequency ratio fopt; (b) $T M D$ damping ratio $\zeta_{2}^{o p t} . \ldots \ldots \ldots \ldots \ldots \ldots$

Percentage reduction of the $\mathrm{H}_{2}$ norm of the displacement of the primary structure at variable mass ratio $\mu$ for different values of structural damping ratio $\zeta_{1}$ : (a) proposed tuning method and (b) Den Hartog tuning method [10]. . . . . . . . . .

$7 \quad$ Primary structure displacement time history with $\zeta_{1}=0$ : (a) proposed tuning method and (b) Den Hartog tuning method [10]. . . . . . . . . . . . . . . . . .

$8 \quad$ Primary structure displacement time history with $\zeta_{1}=0.03$ : (a) proposed tuning method and (b) Den Hartog tuning method [10] . . . . . . . . . . . . . . . . . . .

9 Response displacement time histories $x_{1}(t)$ with $\mu=0.02, \zeta_{1}=0$ for different seismic signals, with passive TMD optimised for unit impulse base displacement.

10 Response displacement time histories $x_{1}(t)$ with $\mu=0.05, \zeta_{1}=0.03$ for different seismic signals, with passive TMD optimised for unit impulse base displacement.

B.1 Scheme of pulse-like excitations adopted in Section 5.2. . . . . . . . . . . . 
Table 1: Percentage reduction of $H_{\infty}$ and $H_{2}$ norms of primary structure displacement $x_{1}(t)$ as a function of different values of mass ratio $\mu$ and structural damping ratio $\zeta_{1}$, achieved with the proposed tuning method.

\begin{tabular}{c|rcc|rrr|rrr}
\hline$\zeta_{1}$ & \multicolumn{3}{|c|}{0} & \multicolumn{3}{|c|}{0.03} & & & \\
$\mu$ & 0.02 & 0.05 & 0.10 & 0.02 & 0.05 & 0.10 & 0.02 & 0.05 & \multicolumn{1}{c}{0.10} \\
\hline$\Delta\left\|x_{1}(t)\right\|_{\infty}[\%]$ & 0.94 & 2.37 & 4.67 & 0.90 & 2.27 & 4.47 & 0.87 & 2.20 & 4.34 \\
$\Delta\left\|x_{1}(t)\right\|_{2}[\%]$ & 62.54 & 70.25 & 75.05 & 28.76 & 38.54 & 46.00 & 19.00 & 27.84 & 35.23 \\
\hline
\end{tabular}


Table 2: Percentage reduction of $H_{\infty}$ and $H_{2}$ norms of primary structure displacement $x_{1}(t)$ as a function of different values of mass ratio $\mu$ and structural damping ratio $\zeta_{1}$, obtained with Den Hartog tuning method [10].

\begin{tabular}{c|rrr|rrr|rrr}
\hline$\zeta_{1}$ & \multicolumn{3}{|c|}{0} & \multicolumn{3}{|c|}{0.03} & & & \\
$\mu$ & 0.02 & 0.05 & 0.10 & 0.02 & 0.05 & 0.10 & 0.02 & 0.05 & \multicolumn{1}{c}{0.10} \\
\hline$\Delta\left\|x_{1}(t)\right\|_{\infty}[\%]$ & 0.95 & 2.40 & 4.70 & 0.92 & 2.31 & 4.52 & 0.89 & 2.22 & 4.41 \\
$\Delta\left\|x_{1}(t)\right\|_{2}[\%]$ & 62.20 & 69.92 & 74.76 & 28.47 & 38.21 & 45.64 & 18.79 & 27.57 & 34.91 \\
\hline
\end{tabular}


Table 3: Average percentage reduction of $H_{\infty}$ and $H_{2}$ norms of primary structure displacement $x_{1}(t)$, with $\mu=0.02, \zeta_{1}=0$, for the four considered pulse-like excitations (Appendix D).

\begin{tabular}{l|l|r|r|r}
\hline \multicolumn{2}{c|}{ Response reduction } & $T_{p}=0.1 T_{1}$ & $T_{p}=0.5 T_{1}$ & $T_{p}=0.8 T_{1}$ \\
\hline \multirow{3}{*}{$\Delta\left\|x_{1}(t)\right\|_{\infty}[\%]$} & Impulse tuning & 0.95 & 1.03 & 0.88 \\
& Den Hartog & 0.96 & 1.02 & 0.87 \\
& $f=1, \zeta_{2}=0.15$ & 1.06 & 1.04 & 0.82 \\
\hline \multirow{3}{*}{$\Delta\left\|x_{1}(t)\right\|_{2}[\%]$} & Impulse tuning & 62.47 & 61.54 & 58.12 \\
& Den Hartog & 62.06 & 61.14 & 57.83 \\
& $f=1, \zeta_{2}=0.15$ & 56.78 & 55.69 & 52.50 \\
\hline
\end{tabular}


Table 4: Average percentage reduction of $H_{\infty}$ and $H_{2}$ norms of primary structure displacement $x_{1}(t)$, with $\mu=0.05, \zeta_{1}=0$, for the four considered pulse-like excitations (Appendix B).

\begin{tabular}{r|l|r|r|r}
\hline \multicolumn{2}{c|}{ Response reduction } & $T_{p}=0.1 T_{1}$ & $T_{p}=0.5 T_{1}$ & $T_{p}=0.8 T_{1}$ \\
\hline \multirow{3}{*}{$\Delta\left\|x_{1}(t)\right\|_{\infty}[\%]$} & Impulse tuning & 2.38 & 2.49 & 2.07 \\
& Den Hartog & 2.41 & 2.46 & 2.02 \\
& $f=1, \zeta_{2}=0.15$ & 2.60 & 2.56 & 2.02 \\
\hline \multirow{3}{*}{$\Delta\left\|x_{1}(t)\right\|_{2}[\%]$} & Impulse tuning & 70.19 & 69.16 & 64.82 \\
& Den Hartog & 69.86 & 68.87 & 64.69 \\
& $f=1, \zeta_{2}=0.15$ & 68.69 & 67.40 & 62.79 \\
\hline
\end{tabular}


Table 5: Average percentage reduction of $H_{\infty}$ and $H_{2}$ norms of primary structure displacement $x_{1}(t)$, with $\mu=0.02, \zeta_{1}=0.03$, for the four considered pulse-like excitations (Appendix B).

\begin{tabular}{l|l|r|r|r}
\hline \multicolumn{2}{c|}{ Response reduction } & $T_{p}=0.1 T_{1}$ & $T_{p}=0.5 T_{1}$ & $T_{p}=0.8 T_{1}$ \\
\hline \multirow{3}{*}{$\Delta\left\|x_{1}(t)\right\|_{\infty}[\%]$} & Impulse tuning & 0.91 & 0.99 & 0.83 \\
& Den Hartog & 0.92 & 0.98 & 0.82 \\
& $f=1, \zeta_{2}=0.15$ & 1.02 & 1.00 & 0.77 \\
\hline \multirow{3}{*}{$\Delta\left\|x_{1}(t)\right\|_{2}[\%]$} & Impulse tuning & 28.75 & 27.92 & 23.64 \\
& Den Hartog & 28.46 & 27.63 & 23.44 \\
& $f=1, \zeta_{2}=0.15$ & 24.69 & 23.77 & 19.92 \\
\hline
\end{tabular}


Table 6: Average percentage reduction of $H_{\infty}$ and $H_{2}$ norms of primary structure displacement $x_{1}(t)$, with $\mu=0.05, \zeta_{1}=0.03$, for the four considered pulse-like excitations (Appendix B).

\begin{tabular}{r|l|r|r|r}
\hline \multicolumn{2}{c|}{ Response reduction } & $T_{p}=0.1 T_{1}$ & $T_{p}=0.5 T_{1}$ & $T_{p}=0.8 T_{1}$ \\
\hline \multirow{3}{*}{$\Delta\left\|x_{1}(t)\right\|_{\infty}[\%]$} & Impulse tuning & 2.28 & 2.38 & 1.95 \\
& Den Hartog & 2.32 & 2.36 & 1.90 \\
& $f=1, \zeta_{2}=0.15$ & 2.50 & 2.45 & 1.89 \\
\hline \multirow{3}{*}{$\Delta\left\|x_{1}(t)\right\|_{2}[\%]$} & Impulse tuning & 38.53 & 37.25 & 31.09 \\
& Den Hartog & 38.19 & 36.94 & 30.95 \\
& $f=1, \zeta_{2}=0.15$ & 36.88 & 35.22 & 28.83 \\
\hline
\end{tabular}


Table 7: Percentage reduction of $H_{\infty}$ and $H_{2}$ norms of primary structure displacement $x_{1}(t)$, with $\mu=0.02$, $\zeta_{1}=0$, for four different earthquake signals, with passive TMD optimised for unit impulse base displacement.

\begin{tabular}{c|rr|rr}
\hline \multirow{2}{*}{ Seismic input } & \multicolumn{2}{|c|}{$\Delta\left\|x_{1}(t)\right\|_{\infty}[\%]$} & \multicolumn{2}{c}{$\Delta\left\|x_{1}(t)\right\|_{2}[\%]$} \\
& Impulse & Den Hartog [10] & Impulse & Den Hartog [10] \\
\hline Imperial Valley, 1940 & 11.43 & 11.27 & 61.78 & 63.18 \\
Loma Prieta, 1989 & 40.32 & 39.75 & 78.93 & 79.28 \\
Kobe, 1995 & 47.93 & 49.30 & 80.90 & 80.45 \\
L'Aquila, 2009 & 29.46 & 28.20 & 77.27 & 77.39 \\
\hline
\end{tabular}


Table 8: Percentage reduction of $H_{\infty}$ and $H_{2}$ norms of primary structure displacement $x_{1}(t)$, with $\mu=0.05$, $\zeta_{1}=0.03$, for four different earthquake signals, with passive TMD optimised for unit impulse base displacement.

\begin{tabular}{c|rr|rr}
\hline \multirow{2}{*}{ Seismic input } & \multicolumn{2}{|c|}{$\Delta\left\|x_{1}(t)\right\|_{\infty}[\%]$} & \multicolumn{2}{c}{$\Delta\left\|x_{1}(t)\right\|_{2}[\%]$} \\
& Impulse & Den Hartog [10] & Impulse & Den Hartog [10] \\
\hline Imperial Valley, 1940 & 10.63 & 10.09 & 30.80 & 30.71 \\
Loma Prieta, 1989 & 22.56 & 21.45 & 17.62 & 21.26 \\
Kobe, 1995 & 8.49 & 10.19 & 31.12 & 32.38 \\
L'Aquila, 2009 & 15.06 & 14.72 & 40.49 & 40.12 \\
\hline
\end{tabular}


Table A.1: Tuning formulae for the optimum TMD parameters as outlined in [38] for different dynamic excitations.

\begin{tabular}{ccc}
\hline Dynamic loading & $f^{\text {opt }}$ & $\zeta_{2}^{\text {opt }}$ \\
\hline Harmonic Force & $1-\sqrt{3 \mu}\left(\frac{1}{2} \sqrt{\mu}+\zeta_{1}\right)$ & $\frac{3}{5} \sqrt{\mu}+\frac{1}{6} \zeta_{1}$ \\
Harmonic Acceleration & $1-\sqrt{3 \mu}\left(\frac{2}{3} \sqrt{\mu}+\frac{3}{2} \zeta_{1}\right)$ & $\frac{3}{5} \sqrt{\mu}+\frac{1}{6} \zeta_{1}$ \\
White Noise Force & $1-\sqrt{3 \mu}\left(\frac{2}{5} \sqrt{\mu}+\frac{1}{4} \zeta_{1}\right)$ & $\frac{1}{2} \sqrt{\mu}$ \\
White Noise Acceleration & $1-\sqrt{3 \mu}\left(\frac{2}{3} \sqrt{\mu}+\frac{3}{2} \zeta_{1}\right)$ & $\frac{1}{2} \sqrt{\mu}$ \\
\hline
\end{tabular}




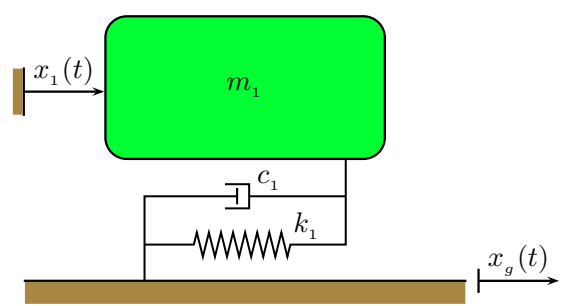

Figure 1: Structural parameters and absolute dynamic degrees of freedom of a SDOF linear primary structure (index 1), subjected to generic base displacement $x_{g}(t)$. 


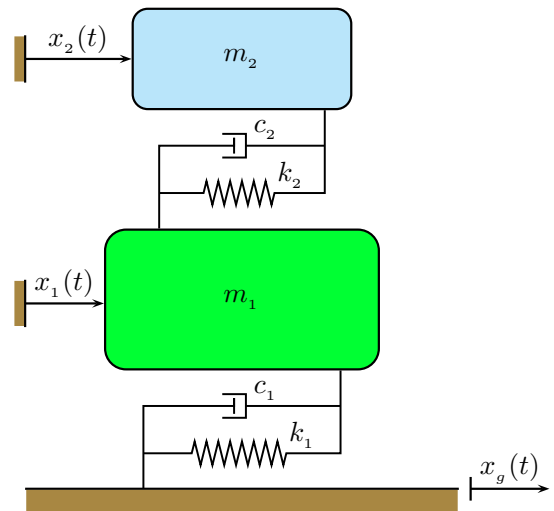

Figure 2: Structural parameters and absolute dynamic degrees of freedom of a 2DOF linear mechanical system comprised of a SDOF primary structure (index 1) equipped with an added TMD (index 2), subjected to generic base displacement $x_{g}(t)$. 


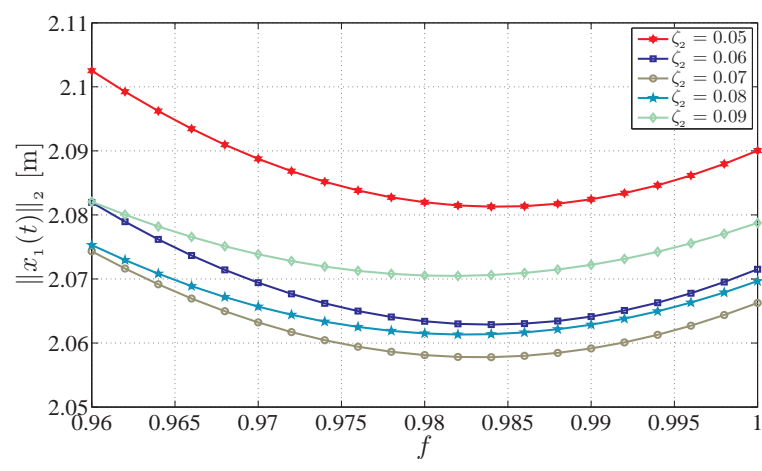

(a) $\mu=0.02$.

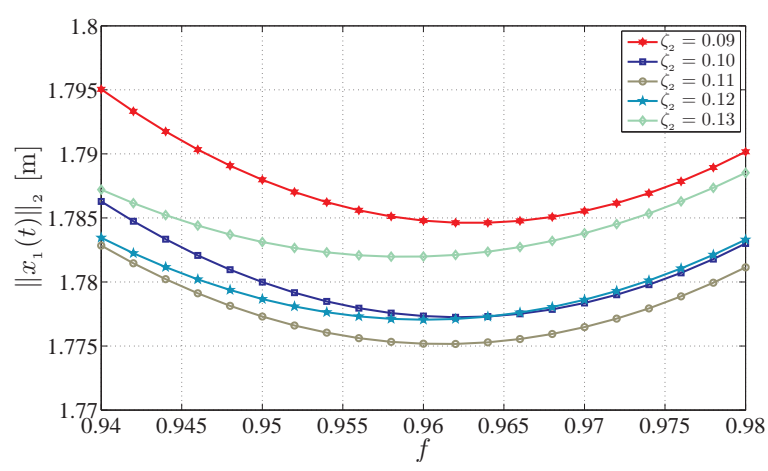

(b) $\mu=0.05$.

Figure 3: $H_{2}$ norm of the primary structure displacement as a function of tuning variables $f, \zeta_{2}$, for $\zeta_{1}=0.03$ and for different values of mass ratio $\mu$. 


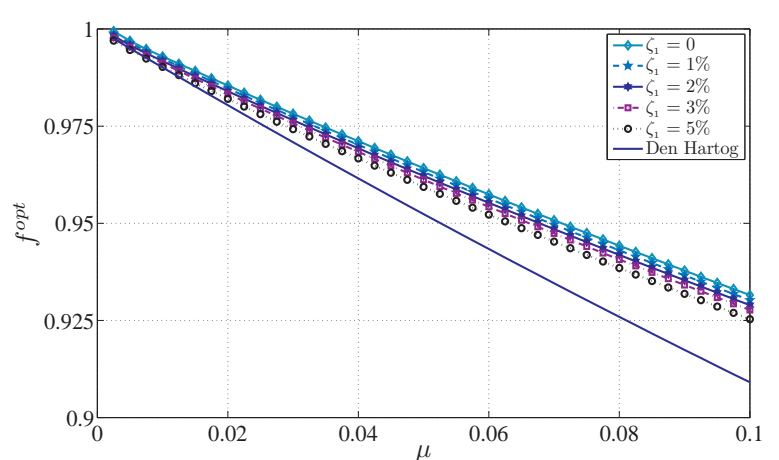

(a)

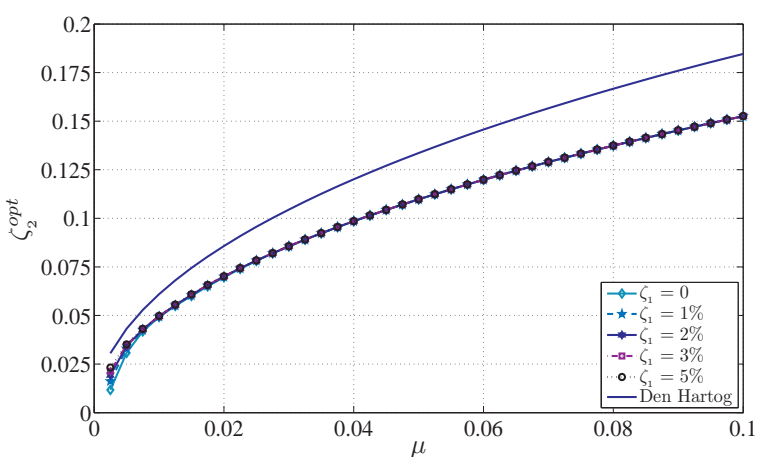

(b)

Figure 4: Optimum TMD parameters at variable mass ratio $\mu$ for different values of structural damping ratio $\zeta_{1}$ : (a) frequency ratio $f^{\text {opt }}$; (b) TMD damping ratio $\zeta_{2}^{\text {opt }}$. 


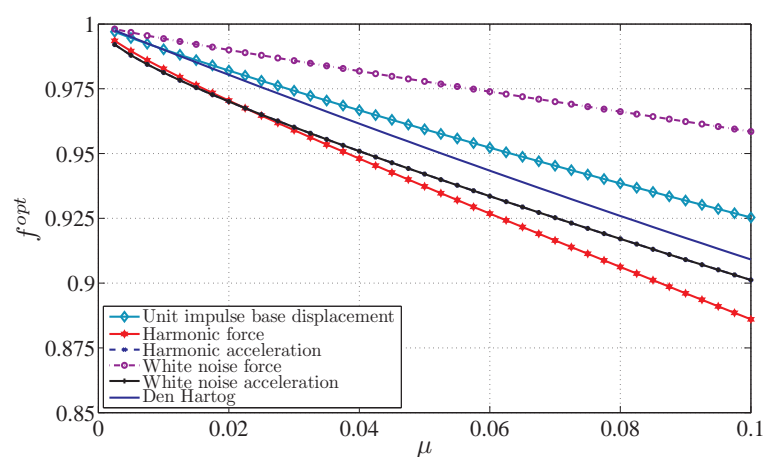

(a)

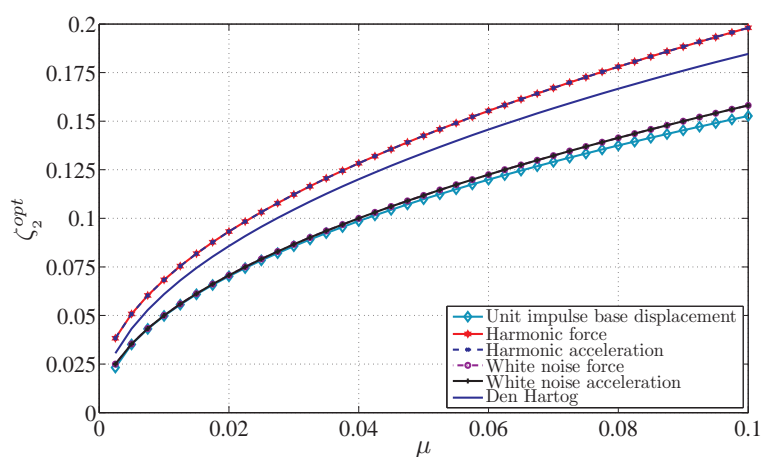

(b)

Figure 5: Optimum TMD parameters at variable mass ratio $\mu$, with structural damping ratio $\zeta_{1}=0.03$, for different dynamic excitations: (a) frequency ratio $f^{\text {opt }}$; (b) TMD damping ratio $\zeta_{2}^{o p t}$. 


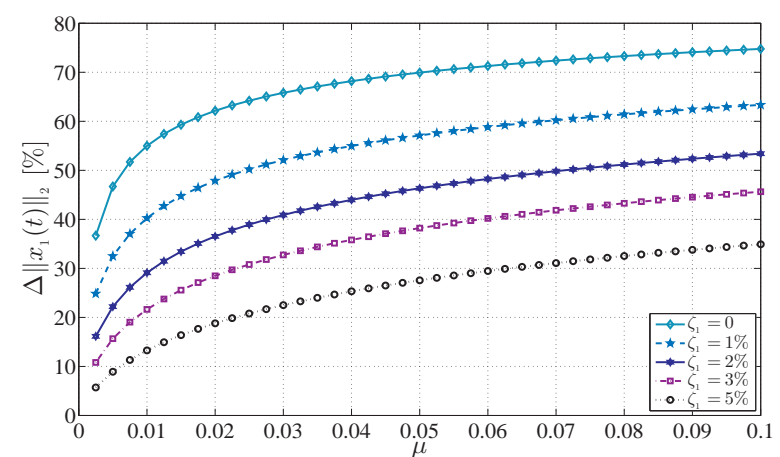

(a)

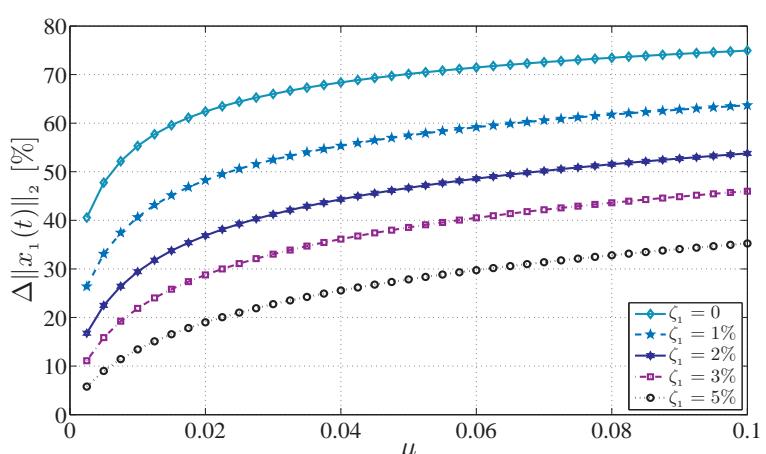

(b)

Figure 6: Percentage reduction of the $\mathrm{H}_{2}$ norm of the displacement of the primary structure at variable mass ratio $\mu$ for different values of structural damping ratio $\zeta_{1}$ : (a) proposed tuning method and (b) Den Hartog tuning method [10]. 


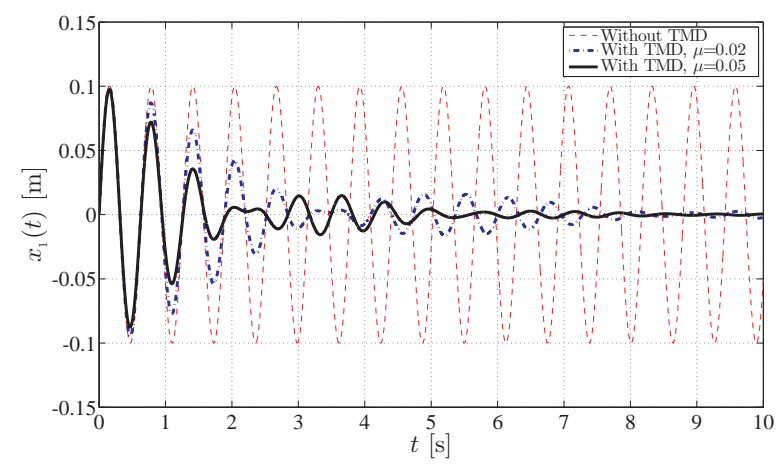

(a)

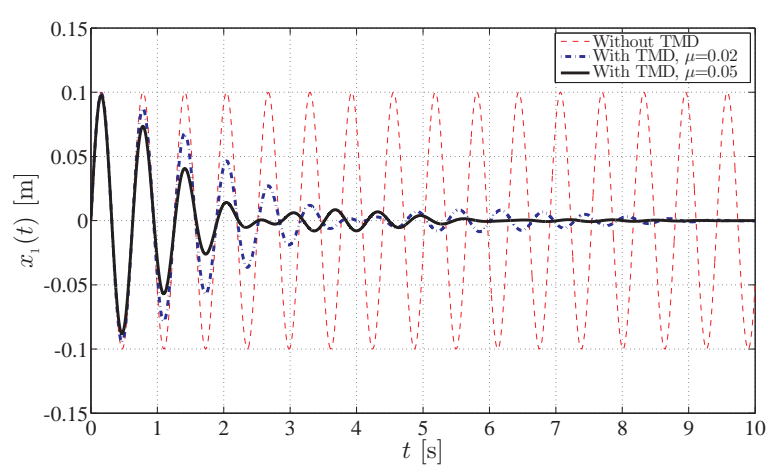

(b)

Figure 7: Primary structure displacement time history with $\zeta_{1}=0:$ (a) proposed tuning method and (b) Den Hartog tuning method [10]. 


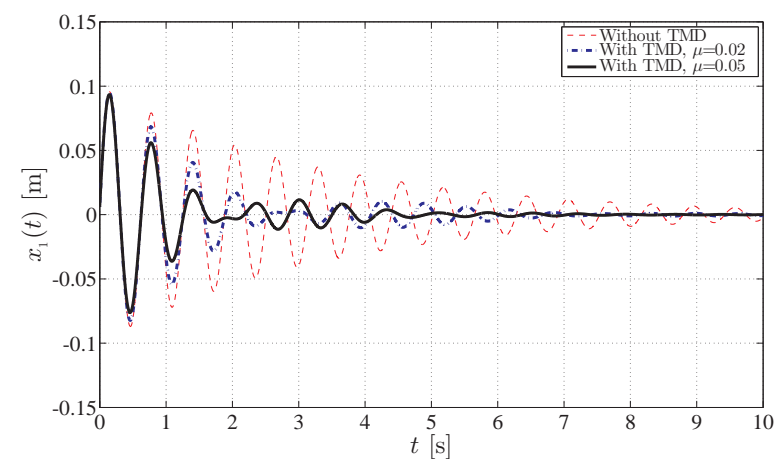

(a)

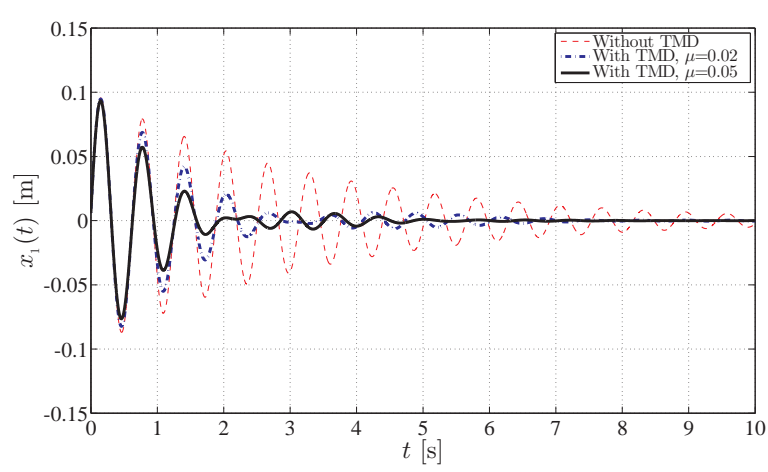

(b)

Figure 8: Primary structure displacement time history with $\zeta_{1}=0.03$ : (a) proposed tuning method and (b) Den Hartog tuning method [10]. 


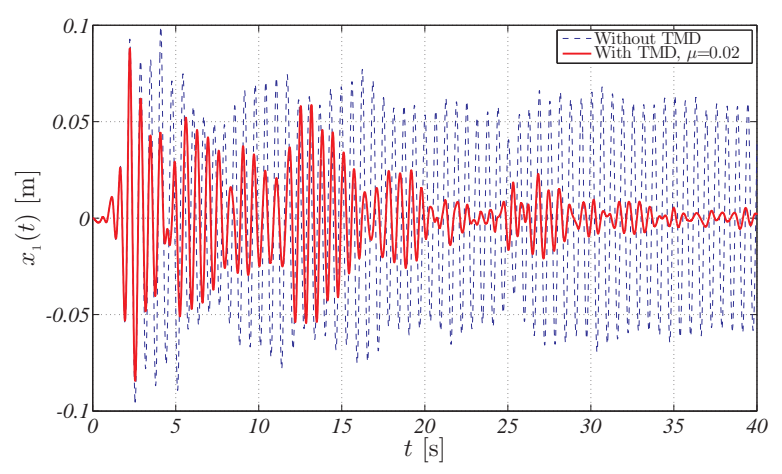

(a) Imperial Valley, 1940.

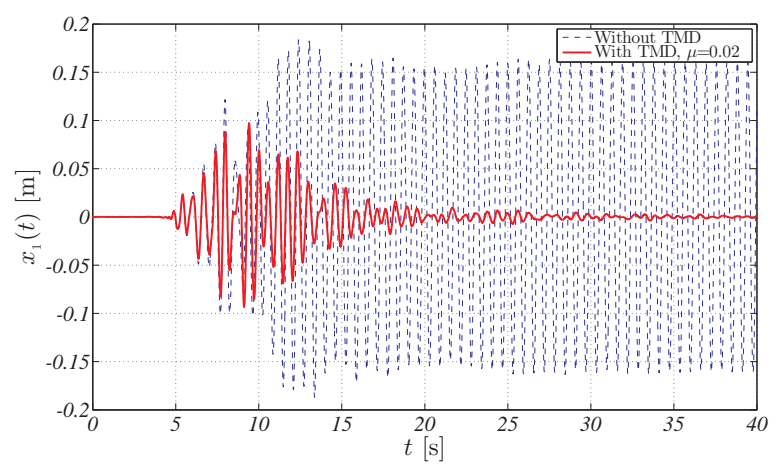

(c) Kobe, 1995 .

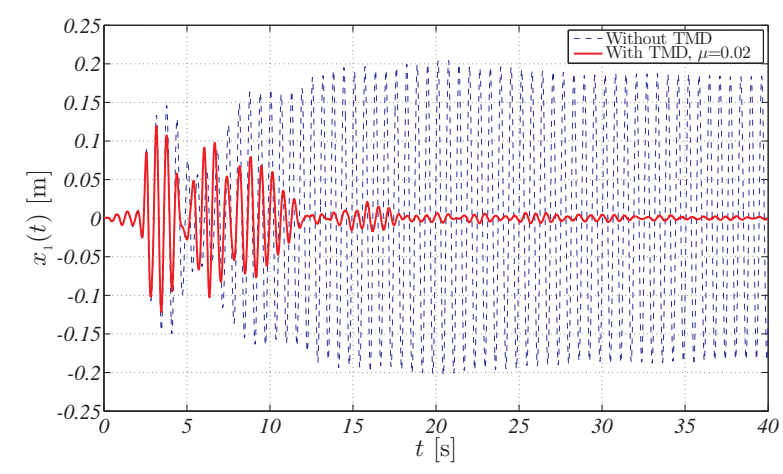

(b) Loma Prieta, 1989.

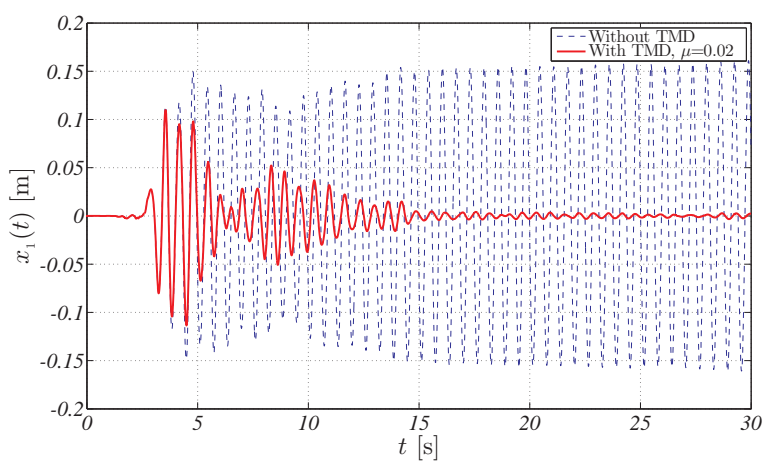

(d) L'Aquila, 2009.

Figure 9: Response displacement time histories $x_{1}(t)$ with $\mu=0.02, \zeta_{1}=0$ for different seismic signals, with passive TMD optimised for unit impulse base displacement. 


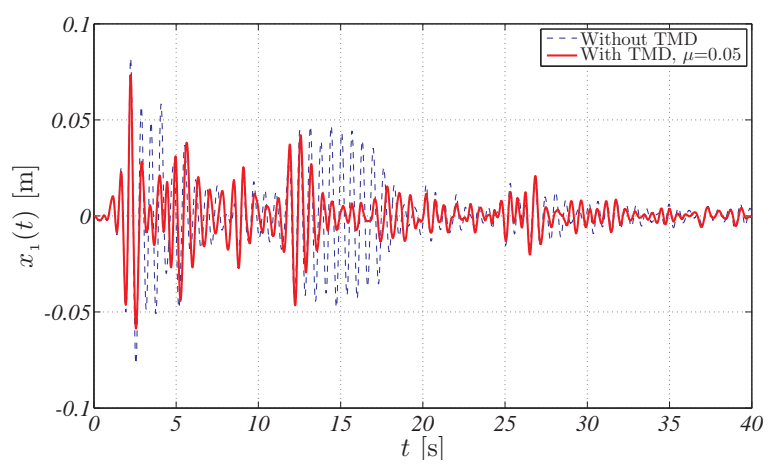

(a) Imperial Valley, 1940.

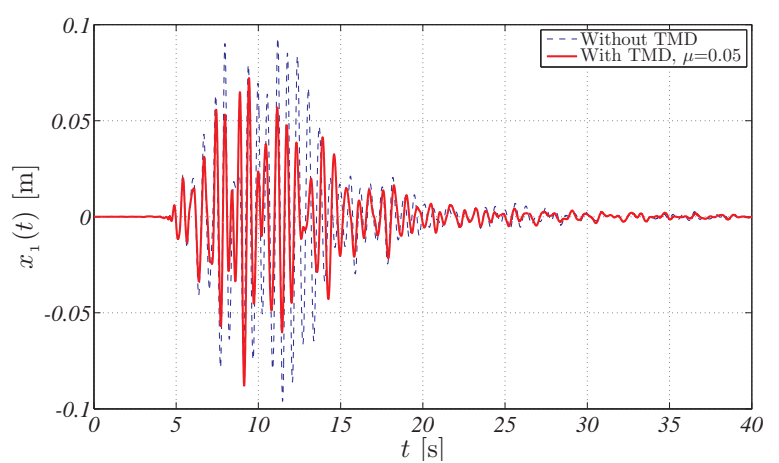

(c) Kobe, 1995 .

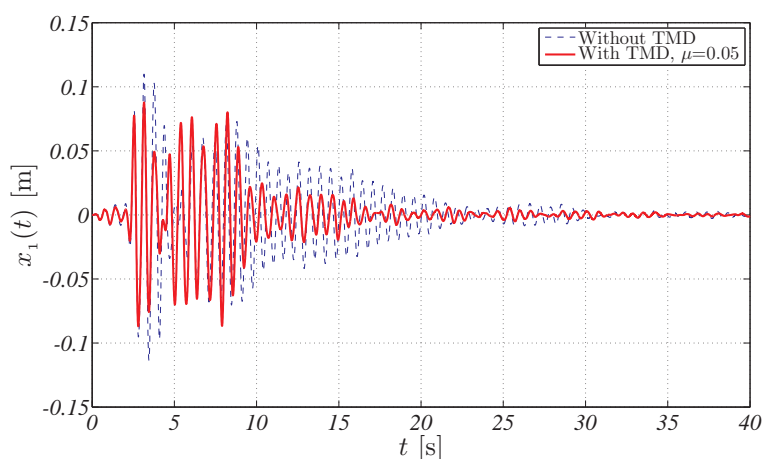

(b) Loma Prieta, 1989.

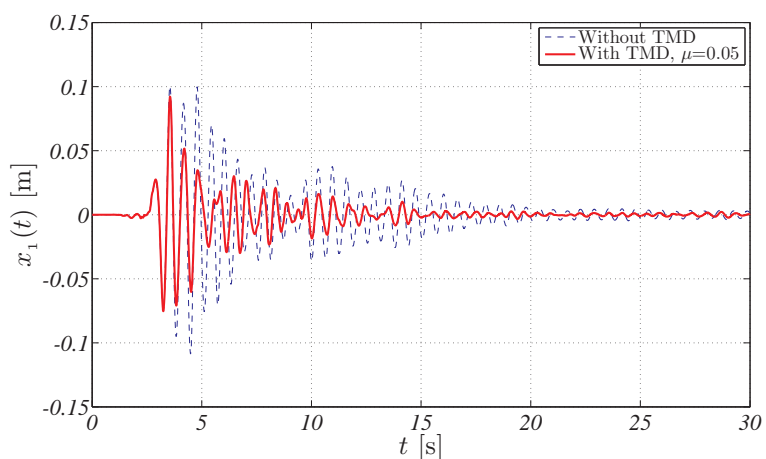

(d) L'Aquila, 2009.

Figure 10: Response displacement time histories $x_{1}(t)$ with $\mu=0.05, \zeta_{1}=0.03$ for different seismic signals, with passive TMD optimised for unit impulse base displacement. 
- Versed-sine pulse: $\bigwedge_{T_{p}} t[\mathrm{~s}]$

$$
\begin{aligned}
x_{g}(t)=\left\{\begin{array}{rl}
\bar{X}_{g} A_{p}\left[1-\cos \left(\omega_{p} t\right)\right], & 0 \leq t \leq T_{p} \\
0, & \text { elsewhere }
\end{array}, \quad A_{p}=\frac{1}{T_{p}}=\frac{\omega_{p}}{2 \pi},\right. \\
X_{g}(s)=\bar{X}_{g} A_{p} \frac{\omega_{p}^{2}\left(1-\mathrm{e}^{-s T_{p}}\right)}{s\left(s^{2}+\omega_{p}^{2}\right)} ; \\
x_{g}(\mathrm{t})[\mathrm{m}]
\end{aligned}
$$

- Linear ramp:

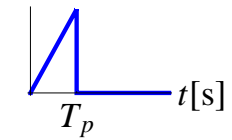

$$
\begin{aligned}
& x_{g}(t)=\left\{\begin{array}{cc}
\bar{X}_{g} A_{p} t, & 0 \leq t \leq T_{p} \\
0, & \text { elsewhere }
\end{array}, \quad A_{p}=\frac{2}{T_{p}^{2}}=\frac{\omega_{p}^{2}}{2 \pi^{2}},\right. \\
& X_{g}(s)=\bar{X}_{g} A_{p} \frac{1-\mathrm{e}^{-s T_{p}\left(1+s T_{p}\right)}}{s^{2}}
\end{aligned}
$$

- Cubic ramp: $\bigwedge_{T_{p}} t[\mathrm{~s}]$

$$
x_{g}(\mathrm{t})[\mathrm{m}]
$$

$$
\begin{aligned}
& x_{g}(t)=\left\{\begin{array}{cc}
\bar{X}_{g} A_{p} \frac{t^{3}}{3}, & 0 \leq t \leq T_{p} \\
0, & \text { elsewhere }
\end{array}, \quad A_{p}=\frac{12}{T_{p}^{4}}=\frac{3 \omega_{p}^{4}}{4 \pi^{4}},\right.
\end{aligned}
$$

- Unit step:

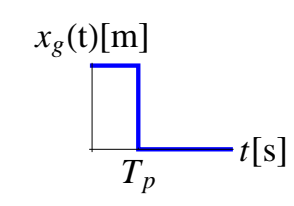

$$
\begin{aligned}
& x_{g}(t)=\left\{\begin{array}{cc}
\bar{X}_{g} A_{p}, & 0 \leq t \leq T_{p} \\
0, & \text { elsewhere }
\end{array}, \quad A_{p}=\frac{1}{T_{p}}=\frac{\omega_{p}}{2 \pi},\right. \\
& X_{g}(s)=\bar{X}_{g} A_{p} \frac{1-\mathrm{e}^{-s T_{p}}}{s} .
\end{aligned}
$$

Figure B.1: Scheme of pulse-like excitations adopted in Section 5.2. 